\title{
Dimensionality Reduction and Classification of Hyperspectral Image Data Using Sequences of Extended Morphological Transformations
}

\author{
Antonio Plaza, Member, IEEE, Pablo Martínez, Javier Plaza, and Rosa Pérez
}

\begin{abstract}
This paper describes sequences of extended morphological transformations for filtering and classification of high-dimensional remotely sensed hyperspectral datasets. The proposed approaches are based on the generalization of concepts from mathematical morphology theory to multichannel imagery. A new vector organization scheme is described, and fundamental morphological vector operations are defined by extension. Extended morphological transformations, characterized by simultaneously considering the spatial and spectral information contained in hyperspectral datasets, are applied to agricultural and urban classification problems where efficacy in discriminating between subtly different ground covers is required. The methods are tested using real hyperspectral imagery collected by the National Aeronautics and Space Administration Jet Propulsion Laboratory Airborne Visible-Infrared Imaging Spectrometer and the German Aerospace Agency Digital Airborne Imaging Spectrometer (DAIS 7915). Experimental results reveal that, by designing morphological filtering methods that take into account the complementary nature of spatial and spectral information in a simultaneous manner, it is possible to alleviate the problems related to each of them when taken separately.
\end{abstract}

Index Terms-Hyperspectral image analysis, morphological filtering, multichannel morphological transformations, neural network classifiers.

\section{INTRODUCTION}

H YPERSPECTRAL remote sensing provides very high spectral resolution image data and the potential for discrimination of subtle differences in ground covers [1], [2]. However, the high-dimensional data space generated by hyperspectral sensors introduces new challenges in the development of data analysis techniques [3], [4]. Previous research has demonstrated that high-dimensional data spaces are mostly empty, indicating that the data structure involved exists primarily in a subspace [5]. As a result, there is a need for feature extraction methods that can reduce the dimensionality of the data to the right subspace without losing the original information that allows for the separation of classes. In other words, dimension reduction is the transformation that brings

Manuscript received February 23, 2004; revised October 17, 2004. This work was supported in part by the European Community within the HySens Project under Contract HPRI-1999-00057 and in part by Junta de Extremadura under Contract 2PR03A026. A. Plaza was supported in part by the Spanish Ministery of Science and Education under a PR2003-0360 Fellowship.

The authors are with the Neural Networks and Signal Processing Group, Computer Science Department, Polytechnic Institute of Cáceres, University of Extremadura, E-10071 Cáceres, Spain (e-mail: aplaza@unex.es; pablomar@unex.es; jplaza@unex.es; rosapere@unex.es).

Digital Object Identifier 10.1109/TGRS.2004.841417 data from a high order dimension to a low order dimension, thus overcoming the "curse" of dimensionality [6].

One of the most widely used dimension reduction techniques in remote sensing is the principal component analysis (PCA). PCA computes orthogonal projections that maximize the amount of data variance, and yields a dataset in a new uncorrelated coordinate system. Unfortunately, information content in hyperspectral images does not always match such projections [7]. This rotational transform is characterized by its global nature, and as a result, it might not preserve all the information useful to obtain a good classification. In order to separate fine-scale from large-scale information, multiscale approaches such as derivative spectroscopy [8] and the wavelet transform [9] have been previously applied to extract relevant features from hyperspectral signals. In derivative spectroscopy, a smoothing operator is followed by a derivative operator to detect "hills" and "valleys" in the spectral curves. More recently, methods using the continuous wavelet transform (CWT) and the discrete wavelet transform (DWT) have been investigated as a means of systematically analyzing hyperspectral curves via windows of varying width. Although the approaches above are particularly suitable for the development of target detection techniques operating at a subpixel level [3], their application to land-cover classification scenarios is limited by the fact that they rely on spectral properties of the data alone, thus neglecting the information related to the spatial arrangement of the pixels in the scene. As a result, feature extraction is carried out without incorporating information on the spatially adjacent data, i.e., the data is managed not as an image but as a disarranged listing of spectral measurements where the spatial coordinates can be randomly shuffled without affecting the analysis [10]. However, one of the distinguishing properties of hyperspectral data, as collected by available imaging spectrometers, is the multivariate information coupled with a two-dimensional (2-D) pictorial representation amenable to image interpretation. Subsequently, there is a need to incorporate the image representation of the data in the development of appropriate feature extraction techniques for the understanding of hyperspectral data.

This paper focuses on the problem of multiscale feature extraction from a broader scope than the individual methods listed above. Instead of focusing exclusively on the spectral information contained in the data, we focus on the analysis of spatial and spectral patterns simultaneously by the design of multichannel filters. Our previous research [11], [12] has examined the application of multichannel mathematical morphology (MM) opera- 
tions to integrate both spatial and spectral responses in hyperspectral data analysis. Classic MM is a nonlinear spatial processing technique [13], [14] that provides a remarkable framework to achieve the desired integration [11]. For perspective, we briefly discuss fundamental operations of classic MM in the context of their application to geoscience and remote sensing problems, and then introduce our multichannel approach.

\section{A. Classic Mathematical Morphology}

Based on set theory, binary MM was established by introducing fundamental operators applied to two sets [15]. One set is processed by another having a carefully selected shape and size, known as the structuring element (SE), which is translated over the image. The SE acts as a probe for extracting or suppressing specific image structures by checking that each position of the SE fits within the image objects. Based on these notions, two fundamental operators are defined in MM, namely erosion and dilation. The application of the erosion operator to an image gives an output image, which shows where the SE fits the objects in the image. In contrast, the application of the dilation operator to an image gives an output image, which shows where the SE hits the objects in the image. All other MM operations can be expressed in terms of erosion and dilation [13]. For instance, the notion behind the opening operator is to dilate an eroded image in order to recover as much as possible of the eroded image. In contrast, the closing operator erodes a dilated image so as to recover the initial shape of image structures that have been dilated. The filtering properties of the opening and closing are based on the fact that, depending on the size and shape of the considered SE, not all structures from the original image will be recovered when these operators are applied [14].

Binary MM operators have been extended to gray-tone (monochannel) images by viewing these data as an imaginary topographic relief; in this regard, the brighter the gray tone, the higher the corresponding elevation [16]. It follows that, in grayscale morphology, each 2-D gray tone image is viewed as if it were a digital elevation model (DEM). In practice, set operators are directly generalized to gray-tone images. For instance, the intersection $\cap$ (respectively, union $U$ ) of two sets becomes the pointwise minimum $\wedge$ (respectively, maximum $\vee$ ) operator [14]. Following a usual notation [17], let us consider a grayscale image $f$, defined on the 2-D discrete space $Z^{2}$, and a SE designed by $B \subset Z^{2}$. The latter is usually "flat" in the sense that it is defined in the spatial domain of the image (the $x-y$ plane) [14]. The flat erosion of $f$ by $B$ is defined by the following expression:

$$
(f \otimes B)(x, y)=\wedge_{(s, t) \in Z^{2}(B)} f(x+s, y+t), \quad(x, y) \in Z^{2}
$$

where $Z^{2}(B)$ denotes the set of discrete spatial coordinates associated to pixels lying within the neighborhood defined by $B$ and $\wedge$ denotes the minimum. In contrast, the flat dilation of $f$ by $B$ is defined by

$$
(f \oplus B)(x, y)=\vee_{(s, t) \in Z^{2}(B)} f(x-s, y-t), \quad(x, y) \in Z^{2}
$$

where $\vee$ denotes the maximum. Using the same notation above, standard opening and closing filters [18] can be, respectively, defined by

$$
\begin{aligned}
& (f \circ B)(x, y)=[(f \otimes B) \oplus B](x, y) \\
& (f \bullet B)(x, y)=[(f \oplus B) \otimes B](x, y), \quad(x, y) \in Z^{2} .
\end{aligned}
$$

The basic operations above have been successfully employed in a variety of geoscience and remote sensing applications [14]. In particular, the idea of using sequences of opening and closing filters to extract adequate features from monochannel remotely sensed data has been widely used by recent research. A composition of geodesic opening and closing operations [18] using SEs of different sizes was used in [19] and [20] in order to build pixel-level differential morphological profiles, which were then used to characterize image structures in high-resolution urban satellite data [21]. Neural-network-based approaches were then used for the classification of the resulting morphological features [21], [22]. More recently, sensor noise-independent morphological profiles were constructed by applying sequences of opening and closing operators alternately, using increasing SE sizes [23]. Extension of the above spatial-based sequential approaches to multichannel imagery has not been fully accomplished yet. An available technique computes the first principal component of the multichannel dataset for use as the base image for constructing morphological profiles by applying monochannel morphological filters [22], [24]. However, extended (multichannel) morphological operations, based on the integration of spatial and spectral properties of the data, should be more effective in discrimination of relevant image features.

\section{B. Multichannel Mathematical Morphology}

The extension of classic MM operations to multichannel image data is not straightforward. When such techniques are applied independently to each image channel (marginal MM), there is a possibility for loss or corruption of information of the image due to the probability that new spectral constituents (not present in the original image) may be created as a result of processing image channels separately [25]. An alternative way to approach the problem of multivariate morphology is to treat the data at each pixel as a vector. In order to define vector MM operations, a concept for a maximum (or minimum) is necessary, and thus it is important to define an appropriate arrangement of vectors in the selected vector space. Since there are no natural means of defining arrangement in $N$-dimensional spaces, a number of vector ordering schemes has been explored [26], [27]. In reduced ordering, a scalar parameter function is computed for each pixel of the image and the ordering is performed according to the resulting scalar values. In partial ordering, the input multivariate samples are partitioned into smaller groups, which are then ordered. In conditional ordering, the vectors are initially ordered according to the ordered values of their components, e.g., the first component. As a second step, vectors that have the same value for the first component are ordered according to the ordered values of another component, e.g., the second component, and so on. Our approach to this problem has been the definition of a reduced vector ordering scheme based on the spectral purity of pixel vectors [11]. First, 
a lattice structure is imposed onto the $N$-dimensional space by ordering input vectors over the values obtained after calculating a spectral-based distance between a certain sample and all the other ordered ones. Second, morphological operations are defined by extension [12].

\section{Scale-Orientation Morphological Profiles (SOMPs)}

One of our main goals in this paper is to incorporate the idea of multiscale analysis into extended MM transformations. In complex image scenes containing structures with several significant derivative maxima and directional features (for instance, urban environments), orientation is worthwhile as an addition to scale information in order to characterize image structures [28], [29]. Morphological directional profiles were defined in [30] by performing openings and closings with line segment SEs of varying orientation [31]. Such profiles were then applied to determine the local orientation of thin elongated structures such as roads on satellite images. Taking advantage of our definition of multichannel morphological transformations, we extend the concepts of differential and scale-orientation morphological profiles to hyperspectral image analysis in this work.

The remainder of the paper is organized as follows. Section II describes the approach followed to extend MM operations to hyperspectral imagery. Section III provides a framework for the calculation of extended morphological sequences, giving various examples. Section IV shows how the above sequences can be used to design a supervised, neural-network-based classification system that is then applied to precision agricultural and urban classification problems. That system is tested using real hyperspectral datasets collected by the Airborne VisibleInfrared Imaging Spectrometer (AVIRIS) and the Digital Airborne Imaging Spectrometer (DAIS 7915) imaging spectrometers. The last section summarizes relevant points made and hints at plausible future research.

\section{EXTENDED MoRPHOLOGICAL TRANSFORMATIONS}

Our attention in this section focuses primarily on the development of a mechanism to extend classic MM filters to hyperspectral image analysis. First, we provide a mathematical formulation for the extension of basic erosion and dilation operations to multichannel imagery. The section ends with an in-depth description of extended opening and closing by reconstruction, a special class of morphological filters that will be used in this work for multiscale image processing.

\section{A. Mathematical Formulation for Extended Dilations and Erosions}

Following the notation in [17], let us consider a hyperspectral image $f$, defined on the $N$-dimensional continuous space $R^{N}$, where $N$ is the number of spectral channels. An ordering relation can be imposed in the set of pixels lying within an SE, denoted by $B$, by defining metrics that calculate the cumulative distance between one particular pixel $\boldsymbol{f}(x, y)$, where $\boldsymbol{f}(x, y)$ denotes an $N$-dimensional vector at discrete spatial coordinates $(x, y) \in Z^{2}$, and every other pixel in the neighborhood given by $B$ [11]. Based on the previous considerations, extended dilation and erosion can, respectively, be defined as follows:

$$
\begin{aligned}
&(\boldsymbol{f} \oplus B)(x, y)=\arg {\left[\vee_{(s, t) \in Z^{2}(B)}\right.} \\
&\left.\left\{\sum_{s} \sum_{t} \operatorname{Dist}(\boldsymbol{f}(x, y), \boldsymbol{f}(x-s, y-t))\right\}\right], \\
&(\boldsymbol{f} \otimes B)(x, y)=\arg [ {\left[\wedge_{(s, t) \in Z^{2}(B)}(x, y) \in Z^{2}(4)\right.} \\
&\left.\left\{\sum_{s} \sum_{t} \operatorname{Dist}(\boldsymbol{f}(x, y), \boldsymbol{f}(x+s, y+t))\right\}\right], \\
&(x, y) \in Z^{2}(5)
\end{aligned}
$$

where Dist is a pointwise distance measure between two $N$-dimensional vectors, and the arg operator, respectively, selects the $N$-dimensional pixel vector that maximizes and minimizes a cumulative distance value between $\boldsymbol{f}(x, y)$ and its neighboring pixels according to $B$. The choice of Dist is a key topic in the resulting ordering relation. For this study, three standard distance measures in hyperspectral analysis are used [3]: spectral angle distance (SAD), spectral information divergence (SID), and hidden Markov model-based information divergence (HMMID). For illustrative purposes, let us consider two $N$-dimensional spectral signatures $\boldsymbol{s}_{i}=\left(s_{i 1}, s_{i 2}, \ldots, s_{i N}\right)^{T}$ and $\boldsymbol{s}_{j}=\left(s_{j 1}, s_{j 2}, \ldots, s_{j N}\right)^{T}$. It is important to emphasize that the term "spectral signature" does not necessarily imply "pixel vector." Subsequently, spatial coordinates are omitted from the two signatures above, although the following argumentation would be the same if two pixel vectors were considered. The $\mathrm{SAD}$ between $\boldsymbol{s}_{i}$ and $\boldsymbol{s}_{j}$ is given by the following expression:

$$
\begin{aligned}
\operatorname{SAD}\left(\boldsymbol{s}_{i}, \boldsymbol{s}_{j}\right) & =\cos ^{-1}\left(\boldsymbol{s}_{i} \cdot \boldsymbol{s}_{j} /\left\|\boldsymbol{s}_{i}\right\|\left\|\boldsymbol{s}_{j}\right\|\right) \\
& =\cos ^{-1}\left(\sum_{l=1}^{N} s_{i l} s_{j l} /\left[\sum_{l=1}^{N} s_{i l}^{2}\right]^{1 / 2}\left[\sum_{l=1}^{N} s_{j l}^{2}\right]^{1 / 2}\right) .
\end{aligned}
$$

It should be noted that the SAD measurement is invariant in the multiplication of the input vectors by constants and, consequently, is invariant to unknown multiplicative scalings that may arise due to differences in illumination and sensor observation angle [32]. In contrast, SID is based on the concept of divergence, and measures the discrepancy of probabilistic behaviors between two spectral signatures. It is based on a process that models $s_{i}$ and $s_{j}$ as a random variables. If we assume that $s_{i l}$ and $s_{j l}, l=1, \ldots, N$, are nonnegative entries, which is generally a valid assumption in remote sensing due to the nature of radiance and reflectance data, then two probability measures can be, respectively, defined for $s_{i}$ and $s_{j}$ as follows:

$$
M\left[s_{i k}\right]=p_{k}=s_{i k} / \sum_{l=1}^{N} s_{i l} \quad M\left[s_{j k}\right]=q_{k}=s_{j k} / \sum_{l=1}^{N} s_{j l} .
$$


Using the above definitions, the self-information provided by $\boldsymbol{s}_{j}$ for band 1 is given by $I_{l}\left(\boldsymbol{s}_{j}\right)=-\log q_{l}$. We can further define the relative entropy of $\boldsymbol{s}_{j}$ with respect to $\boldsymbol{s}_{i}, D\left(\boldsymbol{s}_{i} \| \boldsymbol{s}_{j}\right)$, by [3]

$$
\begin{aligned}
D\left(\boldsymbol{s}_{i} \| \boldsymbol{s}_{j}\right) & =\sum_{l=1}^{N} p_{l} D_{l}\left(\boldsymbol{s}_{i} \| \boldsymbol{s}_{j}\right) \\
& =\sum_{l=1}^{N} p_{l}\left(I_{l}\left(\boldsymbol{s}_{j}\right)-I_{l}\left(\boldsymbol{s}_{i}\right)\right) \\
& =\sum_{l=1}^{N} p_{l} \log \left(p_{l} / q_{l}\right) .
\end{aligned}
$$

By means of (8), a symmetric hyperspectral measure, referred to as SID, is defined as follows:

$$
\operatorname{SID}\left(\boldsymbol{s}_{i}, \boldsymbol{s}_{j}\right)=D\left(\boldsymbol{s}_{i} \| \boldsymbol{s}_{j}\right)+D\left(\boldsymbol{s}_{j} \| \boldsymbol{s}_{i}\right) .
$$

SID offers a new look at the spectral similarity between two spectral signatures by making use of relative entropy, and accounts for the spectral information provided by each signature [33]. Following a similar approach, the HMMID makes use of a hidden Markov model (HMM) to characterize spectral correlation as well as band-to-band variability of hyperspectral image pixel vectors. The HMM process is used to capture the unobserved and hidden spectral properties of the hyperspectral signals. A detailed description of HMMID is available in [34] and will not be discussed here due to similarities with respect to SID and space considerations. Both SID and HMMID are information-theorretic measures that use the concept of self-information [3]. They can be viewed as stochastic measures as opposed to $\mathrm{SAD}$, which is considered to be a deterministic measure.

The use of any of the spectral similarity metrics addressed above as the standard distance measure allows us to impose a partial order relationship of the vectors within a SE in terms of their spectral singularity. It is important to notice that, regardless of the distance metric used in (4) and (5), the proposed operators are vector preserving in the sense that no single vector (constituent) absent from the input data is generated as a result of the extension process.

\section{B. Extended Opening and Closing by Reconstruction Filters}

Extended erosion and dilation allow for the construction of more complex filtering operations with additional properties. The sequential techniques developed in our research are mainly based on opening and closing by reconstruction, a class of morphological filters that do not introduce discontinuities and therefore preserve the shapes observed in input images [35]. Thus, the basic contrast imposed by conventional opening and closing [see (3)] versus reconstruction-based opening and closing can be described as follows. Conventional opening and closing remove the parts of the objects that are smaller than the SE, whereas opening and closing by reconstruction either completely removes the features or retains them as a whole. Following the notation introduced above, extended opening by reconstruction is defined by

$$
(\boldsymbol{f} \circ B)^{k}(x, y)=\vee_{k \geq 1}\left[\delta_{B}^{k}(\boldsymbol{f} \circ B \mid f)\right](x, y)
$$

where

$$
\left[\delta_{B}^{k}(\boldsymbol{f} \circ B \mid \boldsymbol{f})\right](x, y)=\overbrace{\delta_{B} \delta_{B} \cdots \delta_{B}}^{k \text { times }}(\boldsymbol{f} \circ B \mid \boldsymbol{f})](x, y) .
$$

The elementary term $\left[\delta_{B}(\boldsymbol{f} \circ B \mid \boldsymbol{f})\right](x, y)$ is an extended geodesic dilation [36], defined as the pointwise minimum between the elementary dilation of $\boldsymbol{f} \circ B$ using $B$ at pixel $(x, y)$ and the value of $\boldsymbol{f}(x, y)$,

$$
\left[\delta_{B}(\boldsymbol{f} \circ B \mid \boldsymbol{f})\right](x, y)=\wedge\{[(\boldsymbol{f} \circ B) \oplus B](x, y), \boldsymbol{f}(x, y)\} .
$$

As shown in (11), this operation is repeated $\mathrm{k}$ times until stability (i.e., no more pixel value modifications) is achieved [14]. In a similar fashion, extended closing by reconstruction is given by

$$
(\boldsymbol{f} \bullet B)^{k}(x, y)=\wedge_{k \geq 1}\left[\varepsilon_{B}^{k}(\boldsymbol{f} \bullet B \mid \boldsymbol{f})\right](x, y)
$$

where

$$
\left[\varepsilon_{B}^{k}(\boldsymbol{f} \bullet B \mid \boldsymbol{f})\right](x, y)=[\overbrace{\varepsilon_{B} \varepsilon_{B} \cdots \varepsilon_{B}}^{k \text { times }}(\boldsymbol{f} \bullet B \mid \boldsymbol{f})](x, y) .
$$

The elementary term $\left[\varepsilon_{B}(\boldsymbol{f} \bullet B \mid \boldsymbol{f})\right](x, y)$ is an extended geodesic erosion [36], defined as the pointwise maximum between the elementary erosion of $\boldsymbol{f} \bullet B$ using $B$ at pixel $(x, y)$ and the value of $\boldsymbol{f}(x, y)$,

$$
\left[\varepsilon_{B}(\boldsymbol{f} \bullet B \mid \boldsymbol{f})\right](x, y)=\vee\{[(\boldsymbol{f} \bullet B) \otimes B](x, y), \boldsymbol{f}(x, y)\} .
$$

Extended opening and closing by reconstruction will be considered hereinafter as the two letters of our morphological alphabet. These filters demonstrate excellent properties when employed to construct sequential morphological operations for hyperspectral image analysis, in particular, when they are combined with disk-shaped SEs due to their isotropy [22]. Let us denote by $B_{\alpha}^{\text {(square) }}$ a square SE of width $\alpha$ pixels. Similarly, let $B_{\alpha}^{\text {(diamond) }}$ be a diamond-shaped SE with a side of $\alpha$ pixels $(\alpha \geq 2)$. By definition, $B_{1}^{\text {(diamond) }}$ is a single pixel, and $B_{2}^{\text {(diamond) }}$ is the elementary diamond (a pixel and its four direct neighbors). Using the above definitions, disk SEs of increasing radiuses can be approximated by starting with $B_{2}^{\text {(diamond) }}$ and then alternating binary dilations by $B_{3}^{\text {(square) }}$ and $B_{2}^{\text {(diamond) }}$ [14]

$$
\begin{aligned}
B_{\alpha}^{(\text {disk })} & =B_{2}^{\text {(diamond) }} \\
& \times \overbrace{\oplus B_{3}^{\text {(square })} \oplus B_{2}^{\text {(diamond) }} \oplus B_{3}^{\text {(square })} \oplus \cdots}^{\alpha-2 \text { times }}
\end{aligned}
$$

where $\alpha \geq 2$ is the radius in pixels of the approximated disk SE and $\oplus$ is the binary dilation operator. In order to illustrate the geodesic operations in (11) and (14), let $f$ be a hyperspectral image collected by the DLR ROSIS imaging spectrometer [37] over a semiarid area in Cáceres, southwest Spain. The scene consists of 1.2-m $88 \times 134$ pixels, each containing 92 spectral bands covering the spectral range from 504-864 nm. Fig. 1(a) shows the spectral band collected at 584-nm wavelength by the ROSIS imaging spectrometer, where three different materials can be visually identified: cork-oak trees (dark areas), pasture (gray areas), and soil (bright areas). Four target pixels circled in Fig. 1(a) were identified by using an ASD FieldSpec Pro spectroradiometer during a visit to the test site. The pixels in- 


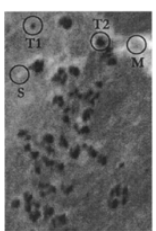

(a)

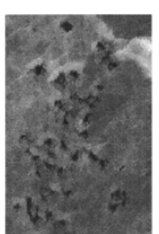

(b)

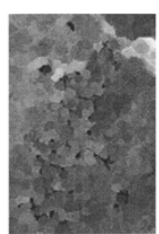

(c)

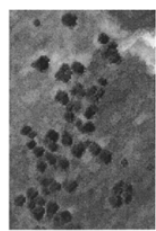

(d)

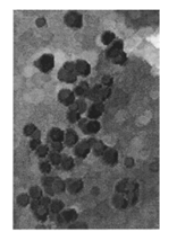

(e)
Fig. 1. Spectral band at $584 \mathrm{~nm}$ of a ROSIS hyperspectral image and spatial positions of four target pixels of interest (a). The same band in the resulting image after applying a geodesic erosion to the original image using (b) $k=1$ and (c) $k=2$ repetitions. The same band in the resulting image after applying a geodesic dilation to the original image using (d) $k=1$ and (e) $k=2$ repetitions.

clude a small cork-oak tree (T1), a medium-sized cork-oak tree (T2), a pure soil area (S), and a mixed area formed by soil and pasture (M). Data collection revealed that T1, T2, and S can be considered spectrally pure at a macroscopic level, while the sensor spatial resolution available does not allow to separate soil from pasture at $\mathrm{M}$, which was labeled as spectrally mixed. Fig. 1(b) and (c), respectively, shows the band at $584 \mathrm{~nm}$ of the resulting image after applying an extended geodesic erosion using $k=1$ and $k=2$ repetitions [see (11)] to the original hyperspectral image in Fig. 1(a). Similarly, Fig. 1(d) and (e), respectively, shows the band at $584 \mathrm{~nm}$ of the resulting image after an extended geodesic dilation using $k=1$ and $k=2$ repetitions [see (14)]. In all cases, the considered SE is given by $B=B_{k+1}^{\text {(disk) }}$. This figure intuitively shows how extended morphological operations affect four pixels with different spectral and spatial properties.

\section{EXTENDED MoRPHOLOGICAL SEQUENCES}

We present below three different approaches to the construction of multiscale sequences of extended MM transformations. Examples illustrating the application of extended morphological sequences to extract relevant features from real hyperspectral datasets are also provided in each subsection.

\section{A. Extended Differential Morphological Profiles}

Morphological profiles for grayscale (monochannel) remotely sensed imagery rely on the sequential application of opening and closing by reconstruction operations using SEs of increasing size [19], [20]. An extended differential morphological profile (EDMP) is defined as a vector where a measure of the spectral variation of the multichannel opening-closing profile is stored for every step of an increasing SE series. Using (10) and (13), we can create EDMPs as follows. Let $M_{k}^{\circ}(x, y)$ be a matrix of dimension $(k+1) \times N$, which contains the opening by reconstruction morphological profile at the point $(x, y)$ of a hyperspectral image $\boldsymbol{f}$, given by

$$
\boldsymbol{M}_{k}^{\circ}(x, y)=\left\{(\boldsymbol{f} \circ B)^{\lambda}(x, y)\right\}, \quad \lambda=\{0,1, \ldots, k\}
$$

where $(f \circ B)^{\lambda}(x, y)$ is the opening by reconstruction operation. Similarly, let $\boldsymbol{M}_{k}(x, y)$ be a matrix of dimension $(k+1) \times N$, which contains the extended closing by reconstruction morphological profile at the point $(x, y)$ of $\boldsymbol{f}$, defined by

$$
\boldsymbol{M}_{k}^{\bullet}(x, y)=\left\{(\boldsymbol{f} \bullet B)^{\lambda}(x, y)\right\}, \quad \lambda=\{0,1, \ldots, k\}
$$

TABLE I

SAD-BASEd LEVEl of THE DERIVATIVE RElative to A SERIES OF OPENING AND ClOSING OPERATIONS FOR SEVERAL PIXELS IN A ROSIS Hyperspectral ScENe OVER a SEMIARID AREA IN SPAIN: T1 (SMAll Cork-OAK Tree), T2 (MEdiuM-Sized Cork-OAK TreE), $\mathrm{S}$ (Pure SoIL) AND M (MiXed PIXEl Formed by SoIL ANd PASture)

\begin{tabular}{ccccccccccccc}
\hline Pixel & $\mathrm{O}_{1}$ & $\mathrm{O}_{2}$ & $\mathrm{O}_{3}$ & $\mathrm{C}_{1}$ & $\mathrm{C}_{2}$ & $\mathrm{C}_{3}$ & $\mathrm{OC}_{1}$ & $\mathrm{OC}_{2}$ & $\mathrm{OC}_{3}$ & $\mathrm{CO}_{1}$ & $\mathrm{CO}_{2}$ & $\mathrm{CO}_{3}$ \\
\hline $\mathrm{T} 1$ & $\mathbf{0 . 2 1 0}$ & 0.014 & 0.007 & 0.002 & 0.001 & 0.001 & $\mathbf{0 . 2 1 0}$ & 0.007 & 0.009 & 0.030 & 0.010 & 0.008 \\
$\mathrm{~T} 2$ & $\mathbf{0 . 1 8 3}$ & 0.003 & 0.001 & 0.030 & 0.002 & 0.001 & $\mathbf{0 . 1 6 0}$ & 0.016 & 0.013 & 0.026 & 0.010 & 0.010 \\
$\mathrm{~S}$ & 0.001 & 0.006 & $\mathbf{0 . 2 4 6}$ & 0.005 & 0.003 & 0.002 & 0.007 & 0.006 & $\mathbf{0 . 1 9 6}$ & 0.008 & 0.014 & 0.026 \\
$\mathrm{M}$ & 0.009 & 0.007 & 0.001 & 0.001 & 0.002 & $\mathbf{0 . 2 1 3}$ & 0.012 & 0.018 & 0.025 & 0.010 & 0.016 & $\mathbf{0 . 1 6 3}$ \\
\hline
\end{tabular}

where $(f \bullet B)^{\lambda}(x, y)$ is the closing by reconstruction operation. Here $(\boldsymbol{f} \bullet B)^{0}(x, y)=\boldsymbol{f}(x, y)=(\boldsymbol{f} \circ B)^{0}(x, y)$ for $\lambda=0$ by the definition of opening and closing by reconstruction [14]. We define the derivative of the extended opening profile, $\boldsymbol{p}_{k}^{\circ}(x, y)$, as the $\mathrm{k}-\mathrm{D}$ vector

$$
\begin{array}{r}
\boldsymbol{p}_{k}^{\circ}(x, y)=\left\{\operatorname{Dist}\left[(f \circ B)^{\lambda}(x, y),(f \circ B)^{\lambda-1}(x, y)\right]\right\}, \\
\lambda=\{1,2, \ldots, k\}
\end{array}
$$

where Dist refers to a spectral-based distance metric such as SAD, SID, or HMMID. By duality, the derivative of the extended closing profile $\boldsymbol{p}_{k}^{\bullet}(x, y)$ is the $\mathrm{k}-\mathrm{D}$ vector

$$
\begin{array}{r}
\boldsymbol{p}_{k}^{\bullet}(x, y)=\left\{\operatorname{Dist}\left[(f \bullet B)^{\lambda}(x, y),(f \bullet B)^{\lambda-1}(x, y)\right]\right\}, \\
\lambda=\{1,2, \ldots, k\} .
\end{array}
$$

For illustrative purposes, Table I shows the level of the derivative relative to a series of opening and closing operations for the target pixels shown in Fig. 1(a), where

$$
\begin{aligned}
O_{\lambda} & =\operatorname{SAD}\left[(f \circ B)^{\lambda}(x, y),(f \circ B)^{\lambda-1}(x, y)\right] \\
C_{\lambda} & =\operatorname{SAD}\left[(f \bullet B)^{\lambda}(x, y),(f \bullet B)^{\lambda-1}(x, y)\right] \\
B & =B_{\lambda+1}^{\text {disk })}
\end{aligned}
$$

and $\lambda=\{1,2,3\}$. As displayed in Table $\mathrm{I}$, pixels that are spectrally pure (T1, T2, and S) show the maximum derivative score in the opening series, while mixed pixels show the maximum derivative score in the closing series. The step of the opening/closing series iteration at which the derivative profile provides a maximum value gives an intuitive idea of both the spectral purity of the pixel and the spatial distribution of the object in the scene. As a result, the derivative profile can be used as a feature vector on which the classification is performed using a spatial/spectral criterion.

\section{B. Extended Alternated Sequential Filters}

Before describing our approach to extend alternated sequential filters (ASFs) to hyperspectral imagery, it is important to note that ASFs rely on reconstruction-based morphological operations [35], [38]. Each opening and closing is followed by a geodesic reconstruction [see (11) and (14)], enabling a perfect preservation of the remaining image features. The sequential application can be based on open-close or close-open filters, i.e., an arbitrary choice must be made on whether to start the sequence with an opening or a closing by reconstruction filter. That choice results in the loss of the morphological self-duality property [23], which was ensured by the EDMP approach (two-sided morphological profile). Let $\boldsymbol{A}_{k}^{\circ}(x, y)$ be a matrix of 
dimension $(k+1) \times N$, which contains the extended open-close alternated sequential filter (EOASF) at the point $(x, y)$ of a hyperspectral image $f$, defined by

$$
A_{k}^{\circ}(x, y)=\left\{[(f \circ B) \bullet B]^{\lambda}(x, y)\right\}, \quad \lambda=\{0,1, \ldots, k\} .
$$

Similarly, let $\boldsymbol{A}_{k}^{\bullet}(x, y)$ be a matrix of dimension $(k+1) \times N$, which contains the extended close-open alternated sequential filter (ECASF) at the point $(x, y)$ of the image $\boldsymbol{f}$, defined by

$$
A_{k}^{\bullet}(x, y)=\left\{[(f \bullet B) \circ B]^{\lambda}(x, y)\right\}, \quad \lambda=\{0,1, \ldots, k\} .
$$

With the above definitions in mind, we should note that both EOASFs and ECASFs could be seen as one-sided morphological profiles, derived by applying alternately opening and closing by reconstruction operators with increasing SE sizes. The derivatives of the EOASF and ECASF are, respectively, given by the $\mathrm{k}-\mathrm{D}$ vectors

$$
\begin{aligned}
& \boldsymbol{q}_{k}^{\circ}(x, y)=\left\{\operatorname{Dist}\left[[(f \circ B) \bullet B]^{\lambda}(x, y),[(\boldsymbol{f} \circ B) \bullet B]^{\lambda-1}(x, y)\right]\right\}, \\
& \lambda=\{1,2, \ldots, k\} \\
& \boldsymbol{q}_{k}^{\bullet}(x, y)=\left\{\operatorname{Dist}\left[[(f \bullet B) \circ B]^{\lambda}(x, y),[(\boldsymbol{f} \bullet B) \circ B]^{\lambda-1}(x, y)\right]\right\}, \\
& \lambda=\{1,2, \ldots, k\} \text {. }
\end{aligned}
$$

For illustrative purposes, Table I shows the level of the derivative relative to a series of alternated openings and closings for the target pixels shown in Fig. 1(a), where

$$
\begin{aligned}
O C_{\lambda} & =\operatorname{SAD}\left[[(\boldsymbol{f} \circ B) \bullet B]^{\lambda}(x, y),[(\boldsymbol{f} \circ B) \bullet B]^{\lambda-1}(x, y)\right] \\
C O_{\lambda} & =\operatorname{SAD}\left[[(\boldsymbol{f} \bullet B) \circ B]^{\lambda}(x, y),[(\boldsymbol{f} \bullet B) \circ B]^{\lambda-1}(x, y)\right] \\
B & =B_{\lambda+1}^{\text {(disk) }}
\end{aligned}
$$

and $\lambda=\{1,2,3\}$. As shown in Table I, open-close filters are particularly useful to discriminate pure spectral features. For instance, the maximum derivative score for pixels $\mathrm{T} 1, \mathrm{~T} 2$, and $\mathrm{S}$ is obtained in the open-close series. In contrast, the maximum derivative score for mixed pixel $\mathrm{M}$ is obtained in the close-open series.

\section{Scale-Orientation Morphological Profiles}

In order to extend the concept of directional morphological profiles to multichannel imagery, we should first denote by $B_{p,(d x, d y)}$ a line segment SE with minimal length, where $\mathrm{p}$ is the number of pixels along the line and $d y / d x$ is the slope of the line segment [31]. By assuming a management of images digitized on a square grid, we can restrict our analysis to line slopes in the form of an irreducible fraction $d y / d x$ (i.e., $d x$ and $d y$ are integers with no common divisors). By convention, we include the forms $0 / 1$ and $1 / 0$ for referring to horizontal and vertical lines, respectively [31]. Let us assume that: 1) a basis set $\mathbf{O}$ contains a collection of $(d x, d y)$ pairs that define the orientations of the considered line segment SE and 2) the resulting line segment SEs are approximated on a discrete grid depending on the length of the line segment [14]. With the above assumptions in mind, we define the SOMP by opening at a given pixel $(x, y)$ of a hyperspectral image $\boldsymbol{f}$ as

$$
\begin{aligned}
\forall(d x, d y) \in \mathbf{O}, & S_{k,(d x, d y)}^{\circ}(x, y) \\
& =\left\{\left[\left(f \circ B_{p,(d x, d y)}\right) \bullet B_{p,(d x, d y)}\right](x, y)\right\}, \\
p & =\{1,2, \ldots, k\} .
\end{aligned}
$$

Similarly, we define the SOMP by closing at the pixel $\boldsymbol{f}(x, y)$ as

$$
\begin{aligned}
\forall(d x, d y) \in \mathbf{O}, \quad S_{k,(d x, d y)}^{\bullet}(x, y) \\
\quad=\left\{\left[\left(f \bullet B_{p,(d x, d y)}\right) \circ B_{p,(d x, d y)}\right](x, y)\right\} \\
p=\{1,2, \ldots, k\} .
\end{aligned}
$$

In both cases, a measure of line strength can be computed for each scale and orientation by calculating the spectral distance (Dist) between the spectral signature in the original image at the pixel $(x, y)$ and the spectral signature at the same location in the image filtered by the considered line segment SE as follows:

$$
\begin{aligned}
& \boldsymbol{r}_{k,(d x, d y)}^{\circ}(x, y)=\{\operatorname{Dist}(\boldsymbol{f}(x, y), \\
& \left.\left.\left[\left(f \circ B_{p,(d x, d y)}\right) \bullet B_{p,(d x, d y)}\right](x, y)\right)\right\} \text {, } \\
& \forall(d x, d y) \in \mathbf{O}, \quad p=\{1,2, \ldots, k\} \quad \text { (27) } \\
& \boldsymbol{r}_{k,(d x, d y)}^{\bullet}(x, y)=\{\operatorname{Dist}(\boldsymbol{f}(x, y), \\
& \left.\left.\left[\left(f \bullet B_{p,(d x, d y)}\right) \circ B_{p,(d x, d y)}\right](x, y)\right)\right\} \text {, } \\
& \forall(d x, d y) \in \mathbf{O}, \quad p=\{1,2, \ldots, k\}
\end{aligned}
$$

where Dist is one of the previously defined SAD, SID or HMMID distance metrics. The resulting values are combined in a feature vector with dimensionality $2 k \times O$, where $O$ is the number of considered orientations. This feature vector can be used for classification purposes. If required by the application under study, the SOMP at a given pixel can be analyzed by plotting the opened and closed values versus the orientation of the line segment by using a polar diagram called "rose of directions" (ROD) [14]. In Fig. 2, the SAD distance was used to construct ROD diagrams associated to four different target object pixels in a DAIS 7915 hyperspectral scene over the city of Pavia, Italy. The scene consists of 5-m $400 \times 400$ pixels, each containing 40 spectral bands covering the spectral range from $496-1756 \mathrm{~nm}$ [22]. Selected target objects include a small building [see Fig. 2(a)], a river [see Fig. 2(b)], a shaded road [see Fig. 2(c)], and a large building [see Fig. 2(d)]. For the sake of simplicity, we consider line segment SEs with $p$ equal to 3, 5, and 7 pixels and four orientations, $\mathbf{O}=\{(1,0),(1,-1),(-1,1),(0,1)\}$. It should be noted that, in the ROD diagrams, the axis denoted by $O_{(d x, d y)}$ (respectively, $\mathrm{C}_{(d x, d y)}$ ) represents line strength values produced by morphological openings (respectively, closings) using a line segment SE with a $d y / d x$ slope. As shown in Fig. 2, directional openings produce a high response with spectrally pure image structures that occur in the direction of the considered line segment SE [see Fig. 2(a) and (d)]. Also, directional closings are appropriate to characterize spectrally mixed image structures in the direction of the considered line segment SE [see Fig. 2(c)]. In both cases, line strength scores are highly 


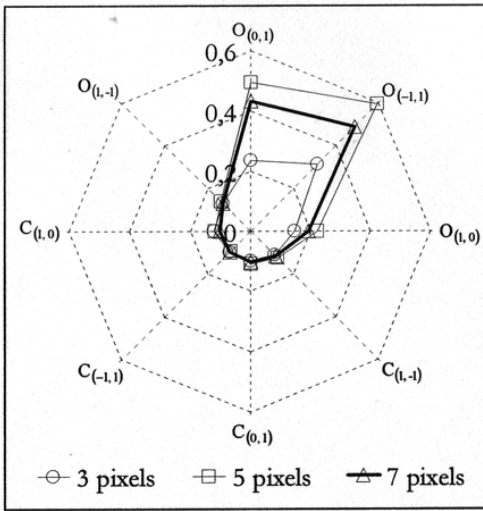

(a)

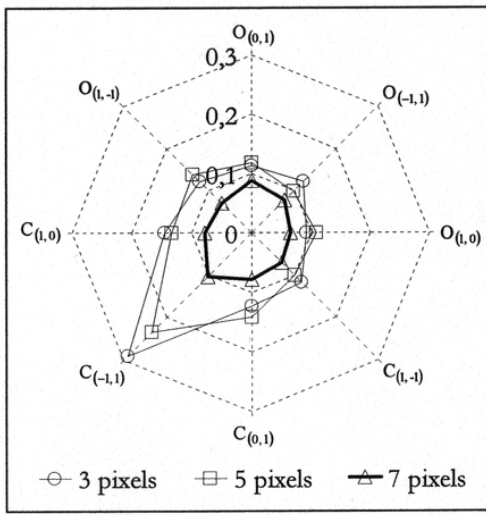

(c)

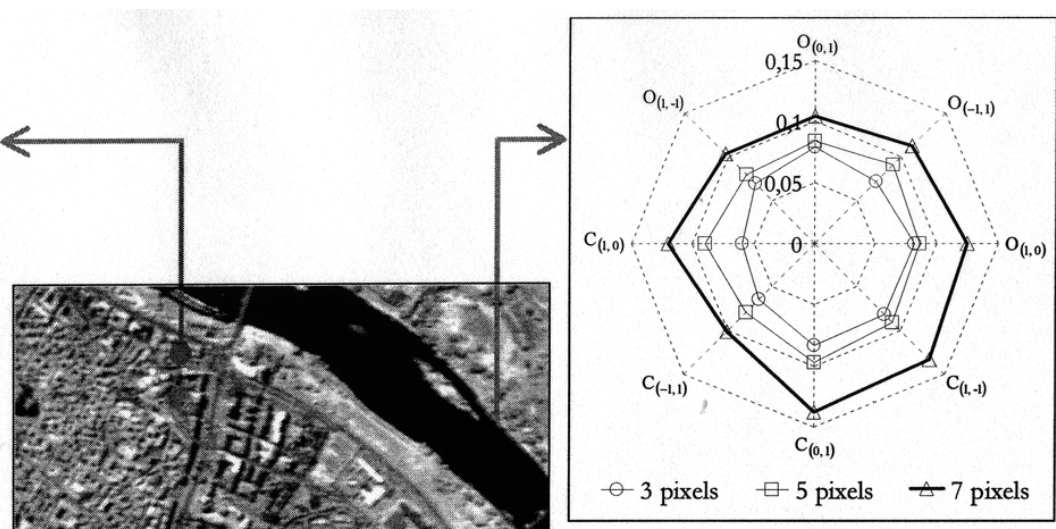

(b)

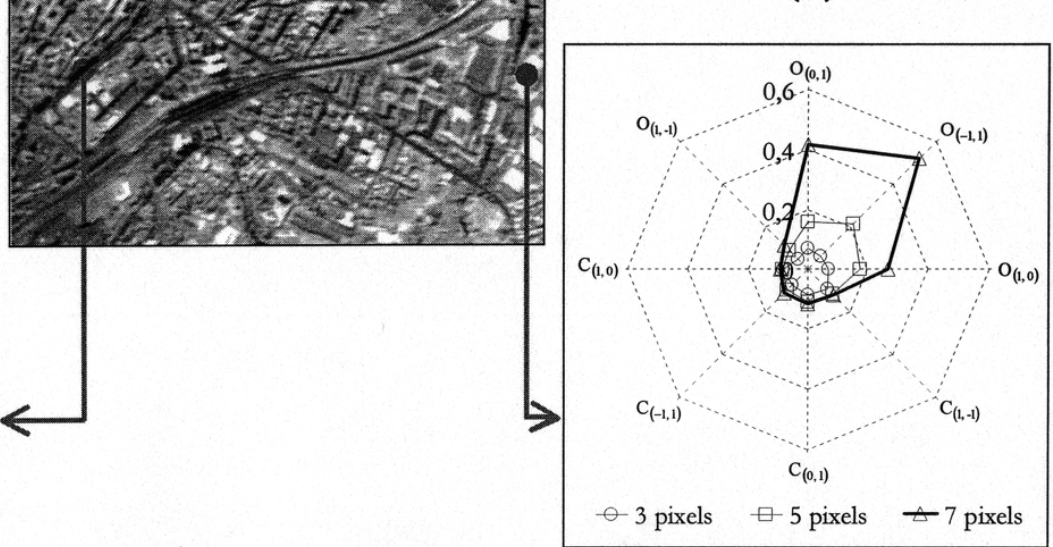

(d)

Fig. 2. Roses of directions showing SOMP-based values of line strength, calculated using line segment SEs with $p=3, p=5$, and $p=7$ pixels in length, for different target objects in a DAIS 7915 hyperspectral scene over Pavia, Italy. (a) Small building. (b) River. (c) Shaded road. (d) Large building.

influenced by the relation between $p$ and the length in pixels of the object. It should be noted that, for pixels belonging to wide (with respect to the line segment SE) objects of similar spectral properties, several distinct orientations might output similar distance values [see Fig. 2(b)]. In the case above, line segment SEs having an increased length may be required in order to accurately characterize the object.

\section{APPLICATIONS}

This section presents two applications of extended morphological transformations focused on the extraction of relevant features for hyperspectral data classification, respectively dominated by agricultural and urban features. In the first application, a portion of an AVIRIS imaging spectrometer dataset taken over an agricultural test site located in Salinas Valley, CA, is used to investigate the accuracy of extended MM sequences in discriminating among several agricultural classes with very similar spectral signatures. In the second application, data collected by the DAIS 7915 airborne imaging spectrometer over the city of Pavia, Italy, were used to test the performance of the morphological filtering techniques above in a more complex scenario dominated by nested regions and directional urban features. In both cases, the proposed analysis system is based on the architecture shown in Fig. 3. First, relevant morphological features are extracted from the original image by using sequences of

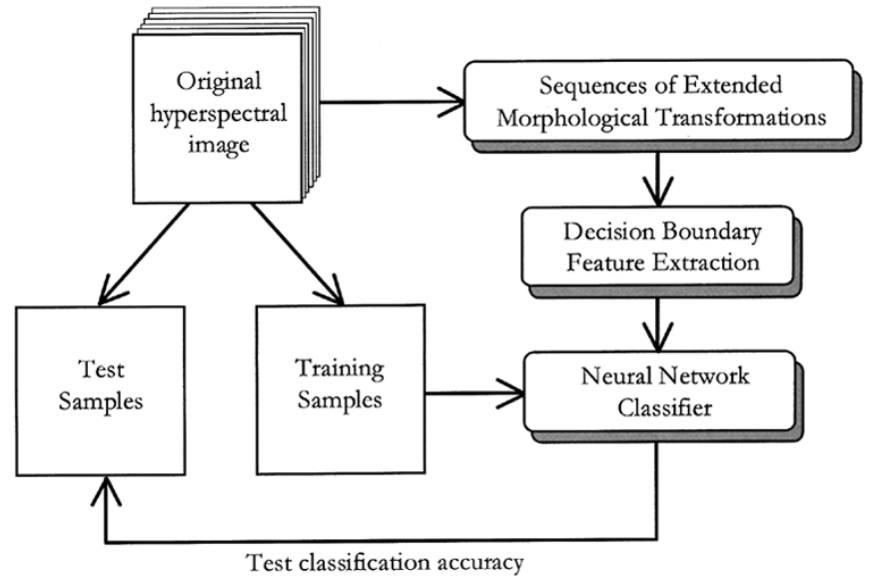

Fig. 3. General architecture of the proposed classification system.

multichannel MM filters. Although morphological filtering usually reduces the dimensionality of the original hyperspectral images, redundancies may still be present in the resulting vectors after the filtering process. Therefore, the application of feature extraction techniques is of great interest to select the most relevant morphological features for class discrimination. In previous literature, Lee and Landgrebe's decision boundary feature extraction (DBFE) [39], [40] has been demonstrated to be a very powerful approach for extracting all the necessary features 


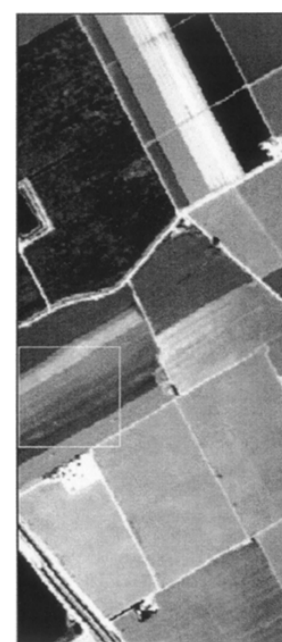

(a)

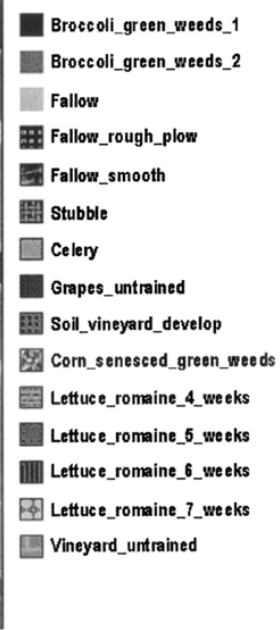$$
\text { (1) }
$$

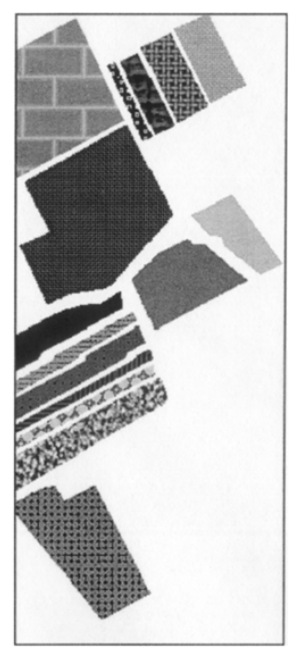

(b)
Fig. 4. (a) Spectral band at $488 \mathrm{~nm}$ of an AVIRIS hyperspectral image comprising several agricultural fields in Salinas Valley, CA, and a subscene of the dataset (Salinas A), outlined by a white rectangle. (b) Land-cover ground truth classes.

for classification of morphological feature vectors [21]. Lee and Landgrebe have extended DBFE for neural networks [41]. This approach has the advantage of preserving the nature of neural networks. Since neural networks can define quite complex decision boundaries without assuming any underlying probability functions, and the DBFE algorithm can find a relevant subspace in a way no other feature extraction method can, they can together provide a helpful insight into the valued applications. In our research, the adopted neural network classifier uses standard backpropagation for supervised learning [4]. This technique can be used to perform efficient nonlinear supervised classification.

\section{A. Experiment 1: AVIRIS Data Over Salinas Valley}

This subsection is a report on the application of the proposed analysis system to real hyperspectral data collected by the AVIRIS imaging spectrometer over Salinas Valley, CA, in 1998. The full scene consists of 512 lines $\times 217$ samples with 192 spectral bands from $0.4-2.5 \mu \mathrm{m}$ after removing the water absorption bands [7], [42], and it was taken at a low altitude with a 3.7-m-pixel size. These data were available only as at-sensor radiance data and include vegetables, bare soils and vineyard fields. Fig. 4(a) shows the entire scene and a subscene of the dataset (called hereinafter Salinas A), which comprises $83 \times 86$ pixels and is dominated by directional features. Fig. 4(b) shows available ground truth regions. Ground truth is available for nearly half of the entire Salinas scene. A random sample of less than $2 \%$ of the pixels was chosen from the known ground truth of the 15 land-cover classes in Fig. 4(b). The resulting number of training and testing pixels for each class are given in Table II. Morphological sequences were constructed for the selected training samples, and DBFE was applied to extract the most relevant features from the sequences above. The resulting features were used to train a backpropagation neural-network-based classifier with one hidden layer, where the number of hidden neurons was selected empirically. The general rule was to select the number of hidden neurons as the square root of the product of the number of input features
TABLE II

INFORMATION CLASSES AND SAMPLES FOR EXPERIMENT 1 (SALINAS AVIRIS SCENE)

\begin{tabular}{lcc}
\hline \hline Class & Training Samples & Test Samples \\
\hline Broccoli_green_weeds_1 & 40 & 1893 \\
Broccoli_green_weeds_2 & 43 & 3704 \\
Fallow & 41 & 1960 \\
Fallow_rough_plow & 38 & 697 \\
Fallow_smooth & 47 & 1349 \\
Stubble & 48 & 1677 \\
Celery & 44 & 1926 \\
Grapes_untrained & 60 & 10305 \\
Soil_vineyard_develop & 58 & 6128 \\
Corn_senesced_green_weeds & 55 & 3154 \\
Lettuce_romaine_4_weeks & 44 & 984 \\
Lettuce_romaine_5_weeks & 47 & 1850 \\
Lettuce_romaine_6_weeks & 42 & 818 \\
Lettuce_romaine_7_weeks & 39 & 1003 \\
Vineyard_untrained & 49 & 7055 \\
\hline Total & 695 & 44503 \\
\hline \hline
\end{tabular}

and information classes [22]. However, in all cases, several configurations of the hidden layer were tested and the one that gave the higher overall accuracies was reported. In the two considered experiments, the best performance was generally observed by selecting the number of hidden neurons as twice the number of input features. The trained classifier was then applied to the remaining $98 \%$ of the known ground truth pixels in the scene, yielding the results depicted in Fig. 5 for a variety of input morphological sequences.

In order to ensure the fairest possible comparison between the different feature extraction approaches tested, it is important to find the most appropriate parameter values for each method. Then, prior to a full examination and discussion of the results, it is important to outline parameter values used for the construction of morphological sequences. Fig. 5(a)-(c), respectively, plots the overall test classification accuracies, obtained after combining the DBFE-based system with multichannel EDMP, EOASF, and ECASF sequences, as a function of the number of opening/closing operations. For comparative purposes, standard differential morphological profiles (DMPs), open-close alternated sequential filters (OASFs), and close-open alternated sequential filters (CASFs) were also constructed. These methods are equivalent to EDMP, EOASF, and ECASF, respectively, but they are based on monochannel morphological processing of the first principal component. It should be noted that, according to our experiments, the use of other widely used component transformations such as the maximum noise fraction or singular value decomposition does not have a significant impact on the performance of DMP, OASF, and CASF methods. Three different distance metrics (SAD, SID, and HMMID) were used for the development of EDMP, EOASF, and ECASF sequences, while the base distance for the construction of DMP, OASF, and CASF sequences was the Euclidean distance (ED). The 3-D graphs in Fig. 5(d)-(f) plot test accuracies, produced by using SOMP-based morphological sequences, as a function of both the length in pixels and the number of directions considered in the design of line segment 


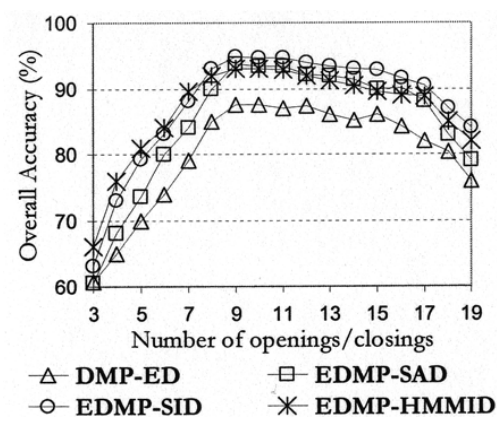

(a)

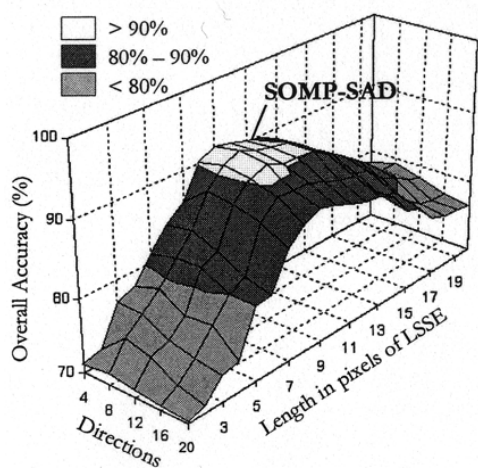

(d)

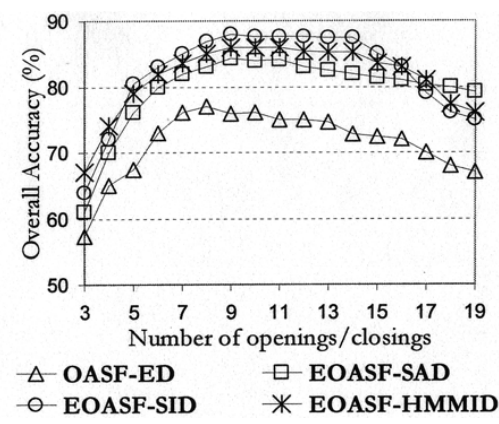

(b)

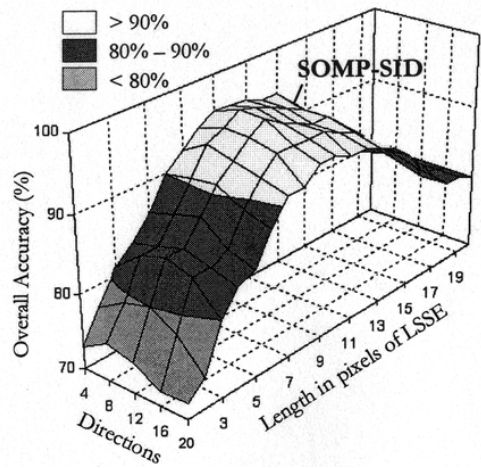

(e)

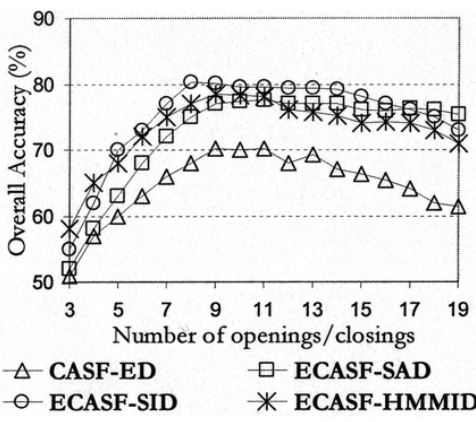

(c)

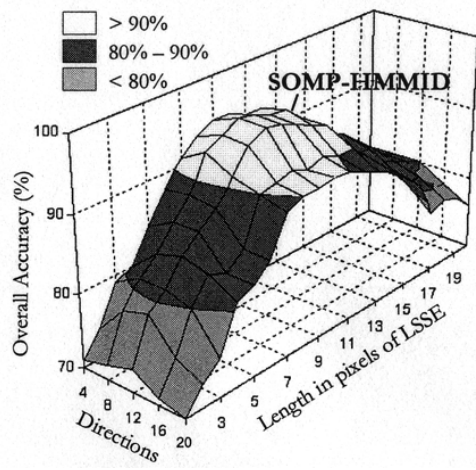

(f)

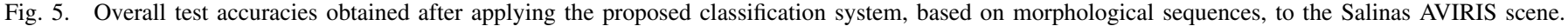
(a) DMP- and EDMP-based sequences. (b) OASF- and EOASF-based sequences. (c) CASF- and ECASF-based sequences. (d)-(f) SOMP-based sequences.

SEs. Again, the SAD [Fig. 5(d)], SID [Fig. 5(e)], and HMMID [Fig. 5(f)] distance metrics were considered for the construction of the sequences.

From Fig. 5(a)-(c), it is clear that the pixelwise width of interesting patterns in the Salinas AVIRIS scene makes nine opening/closing iterations a reasonable parameter selection for both multi and monochannel morphological methods tested in this experiment (the number of openings is always equal to the number of closings). In particular, the best overall accuracies in Fig. 5(a) were achieved when EDMP sequences were constructed using the SID distance (EDMP-SID hereinafter). Both EDMP-HMMID and EDMP-SAD performed slightly better than DMP-ED. It should be noted that EOASF-SID also produced slightly better results than EOASF-SAD and EOASF-HMMID [see Fig. 5(b)], while ECASF-SID improved both ECASF-SAD and ECASF-HMMID [see Fig. 5(c)]. This fact points out that SID has a better ability than HMMID and SAD in characterizing spectral properties of land-cover classes in this particular scene. However, DMP-ED produced clearly better results than all EOASF- and ECASF-based approaches, as shown in Fig. 5(b) and (c). This confirms the effectiveness of two-sided morphological profiles with regards to one-sided alternate filters in this example. Finally, the higher accuracies for SOMP-based sequences were also found when the SID distance was used [Fig. 5(e)]. A general trend observed in Fig. 5(d)-(f) is that SOMP series produced the best classification scores when line segment SEs having nine pixels in length and eight equidistant directions were used. As a result, our final choice for the construction of scale-orientation sequences in this experiment was eight directions and $p=9$. In general, the best results for accuracy assessments were obtained from utilizing a moderate number of scales and orientations. The construction of morphological feature vectors with very large data dimensions generally caused a loss in classification performance.

With the above parameter settings in mind, the dimension of feature vectors after morphological filtering, number of features used for classification after DBFE feature extraction, overall (OA), average (AVE) and individual test accuracies in percentage exhibited by the aforementioned classifiers on the Salinas dataset are given in Table III. For comparative purposes, classification accuracies obtained using the original spectral information in the hyperspectral image are also reported. The $\mathrm{OA}$ and AVE accuracies were calculated as follows. Let us assume that $P$ ground truth categories $C_{1}, \ldots, C_{P}$ are given, and spectrum data of $n_{i}$ test samples from the categories $C_{i}$ are observed for $i=1, \ldots, P$. We classify all test samples into one of the categories by a certain classification method. Let the term $a_{i j}$ represent the number of test samples that actually belong to class $C_{i}$ and are classified into $C_{j}$, for $i, j=1, \ldots, P$. The row total $\sum_{j=1}^{P} a_{i j}$ is equal to the sample number $n_{i}$ from $C_{i}$, and the column total $\sum_{i=1}^{P} a_{i j}$ denotes the number of samples classified into $C_{j}$. Let $N=\sum_{i=1}^{P} n_{i}$ be the total number of samples. The following coefficients for accuracy assessment are used in this work:

$$
\mathrm{OA}=\frac{1}{N} \sum_{i=1}^{P} a_{i i} \quad \mathrm{AVE}=\frac{1}{P} \sum_{i=1}^{P} a_{i i} / n_{i}
$$

As shown in Table III, DBFE did not require many features to produce high test accuracies. As a result, it was possible, in 
TABLE III

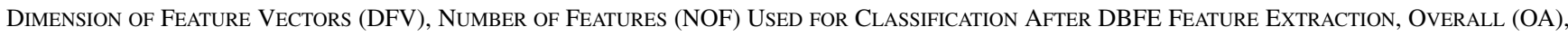
Average (AVE), and Individual Test Accuracies in Percentage ObTained After ApPlying the Proposed Classification System, Based on Morphological Sequences With Nine Opening/Closing Operations, to the Salinas AVIRIS SCENE (SOMP-BASED FEATURES Were ObTained By ApPlying Line SEgment Structuring ElEMENTS With LENGTH of Nine PiXels and Eight ORIENTATIONS). Classification Results ObTAINED USING THE ORIGINAL SPECTRAL INFORMATION OF THE HYPERSPECTRAL SCENE ARE ALSO INCLUDED FOR COMPARISON

\begin{tabular}{|c|c|c|c|c|c|c|c|c|c|c|c|c|c|c|c|c|}
\hline $\begin{array}{l}\text { Class } \\
\left({ }^{*} \text { denotes Salinas A classes }\right)\end{array}$ & $\begin{array}{c}\text { Original } \\
\text { Spectral } \\
\text { Information }\end{array}$ & $\begin{array}{c}\text { DMP- } \\
\text { ED }\end{array}$ & $\begin{array}{l}\text { EDMP- } \\
\text { SAD }\end{array}$ & $\begin{array}{l}\text { EDMP- } \\
\text { SID }\end{array}$ & $\begin{array}{l}\text { EDMP- } \\
\text { HMMID }\end{array}$ & $\begin{array}{c}\text { OASF- } \\
\text { ED }\end{array}$ & $\begin{array}{l}\text { EOASF- } \\
\text { SAD }\end{array}$ & $\begin{array}{l}\text { EOASF- } \\
\text { SID }\end{array}$ & $\begin{array}{l}\text { EOASF- } \\
\text { HMMID }\end{array}$ & $\begin{array}{l}\text { CASF- } \\
\text { ED }\end{array}$ & $\begin{array}{l}\text { ECASF- } \\
\text { SAD }\end{array}$ & $\begin{array}{l}\text { ECASF- } \\
\text { SID }\end{array}$ & $\begin{array}{l}\text { ECASF- } \\
\text { HMMID }\end{array}$ & $\begin{array}{l}\text { SOMP- } \\
\text { SAD }\end{array}$ & $\begin{array}{l}\text { SOMP- } \\
\text { SID }\end{array}$ & $\begin{array}{l}\text { SOMP- } \\
\text { HMMID }\end{array}$ \\
\hline Broccoli_green_weeds_1* & 78.42 & 79.03 & 81.25 & 82.75 & 82.03 & 72.25 & 77.79 & 76.21 & 77.31 & 67.53 & 74.12 & 75.23 & 74.71 & 90.34 & 92.31 & 91.49 \\
\hline Broccoli_green_weeds_2 & 80.13 & 81.27 & 83.02 & 86.21 & 84.58 & 70.69 & 79.42 & 81.42 & 80.29 & 66.48 & 72.97 & 75.76 & 73.65 & 90.05 & 91.45 & 90.19 \\
\hline Fallow & 92.98 & 95.38 & 96.59 & 98.08 & 96.73 & 83.04 & 92.11 & 93.74 & 93.25 & 78.02 & 84.76 & 86.52 & 85.01 & 97.11 & 98.24 & 97.89 \\
\hline Fallow_rough_plow & 96.51 & 92.27 & 94.52 & 96.42 & 95.01 & 82.77 & 89.34 & 92.15 & 91.92 & 77.69 & 84.65 & 85.28 & 84.72 & 94.89 & 96.89 & 96.45 \\
\hline Fallow_smooth & 93.72 & 92.79 & 95.01 & 97.62 & 96.35 & 81.96 & 89.20 & 93.87 & 93.24 & 76.21 & 84.79 & 86.34 & 85.21 & 96.12 & 97.91 & 97.94 \\
\hline Stubble & 94.71 & 95.35 & 98.02 & 98.12 & 97.89 & 82.44 & 91.16 & 95.12 & 95.49 & 76.94 & 83.21 & 86.13 & 84.89 & 97.89 & 98.02 & 97.35 \\
\hline Celery & 89.34 & 93.47 & 99.05 & 98.10 & 99.12 & 82.23 & 93.27 & 95.03 & 94.18 & 78.05 & 84.46 & 85.45 & 83.28 & 98.17 & 98.24 & 98.31 \\
\hline Grapes_untrained & 88.02 & 90.57 & 93.78 & 95.25 & 94.16 & 78.46 & 83.94 & 87.16 & 86.28 & 73.84 & 81.06 & 82.32 & 81.46 & 95.03 & 95.43 & 94.27 \\
\hline Soil_vineyard_develop & 88.55 & 88.41 & 89.13 & 90.31 & 89.44 & 75.86 & 82.93 & 84.76 & 84.25 & 70.35 & 78.24 & 79.73 & 79.94 & 91.24 & 93.02 & 92.79 \\
\hline Corn_senesced_green_weeds* & 87.46 & 84.08 & 83.90 & 82.21 & 82.29 & 66.49 & 74.68 & 76.48 & 75.27 & 62.03 & 67.70 & 69.69 & 68.72 & 89.36 & 91.32 & 90.12 \\
\hline Lettuce_romaine_4_weeks* & 78.86 & 81.43 & 82.28 & 83.12 & 83.10 & 68.36 & 77.91 & 76.82 & 76.41 & 63.86 & 71.39 & 72.66 & 71.19 & 88.21 & 90.87 & 89.45 \\
\hline Lettuce_romaine_5_weeks* & 91.35 & 78.14 & 79.28 & 82.03 & 80.57 & 69.13 & 76.35 & 78.23 & 77.63 & 65.24 & 71.28 & 73.18 & 71.94 & 87.93 & 89.49 & 88.76 \\
\hline Lettuce_romaine_6_weeks* & 88.53 & 79.11 & 81.81 & 84.18 & 82.25 & 69.98 & 78.20 & 77.84 & 78.12 & 64.89 & 72.46 & 75.44 & 74.83 & 90.23 & 92.07 & 91.65 \\
\hline Lettuce_romaine_7_weeks* & 84.85 & 81.27 & 84.23 & 86.43 & 85.52 & 76.12 & 80.11 & 81.52 & 81.32 & 72.21 & 76.54 & 77.15 & 77.03 & 91.94 & 94.12 & 93.48 \\
\hline Vineyard_untrained & 87.14 & 87.95 & 91.27 & 92.79 & 91.07 & 75.28 & 84.07 & 85.67 & 84.69 & 70.13 & 77.41 & 78.86 & 77.25 & 93.67 & 94.26 & 94.69 \\
\hline DFV & 192 & 18 & 18 & 18 & 18 & 18 & 18 & 18 & 18 & 18 & 18 & 18 & 18 & 144 & 144 & 144 \\
\hline NOF & 15 & 6 & 7 & 8 & 7 & 5 & 9 & 8 & 8 & 6 & 7 & 8 & 8 & 13 & 14 & 14 \\
\hline OA & 87.55 & 88.21 & 93.85 & 94.12 & 93.08 & 75.83 & 84.31 & 87.89 & 86.27 & 70.89 & 77.05 & 80.23 & 78.49 & 94.34 & 95.27 & 95.03 \\
\hline AVE & 88.03 & 86.67 & 88.93 & 90.66 & 89.34 & 75.67 & 83.36 & 85.07 & 84.64 & 70.16 & 77.66 & 79.29 & 78.32 & 92.81 & 94.24 & 93.65 \\
\hline
\end{tabular}

most cases, to keep the number of features very low without sacrificing OA and AVE scores. As can be seen, the OAs exhibited by both EDMP- and SOMP-based approaches are very high for the three distance measures (i.e., $93.85 \%$, and $94.34 \%$ for the SAD, $94.12 \%$ and $95.27 \%$ for the SID, and $93.08 \%$ and $95.03 \%$ for the HMMID). These scores are sensibly higher than the accuracy obtained using the original spectral information of the image (i.e., $87.55 \%$ ). It is important to note that the dimension of feature vectors (DFV) of the original spectral information in Table III is 192. Since the network was trained using 695 samples, the performance of the baseline method suffers from limited training samples. However, we observed, that even when the number of training samples was increased to 22600 (i.e., half of the available ground-truth), the results obtained by the classifier based on the original spectral information could not improve those found by EDMP- and SOMP-based approaches using only 695 training samples, as pointed out below. Finally, the OAs provided by EOASF- and ECASF-based approaches (i.e., $84.31 \%$, and $77.05 \%$ for the SAD, $87.89 \%$, and $80.23 \%$ for the SID, and $86.27 \%$ and $78.49 \%$ for the HMMID) are all lower than the OA produced by DMP-ED (i.e., 88.21\%).

Comparisons between multichannel sequential filters (i.e., EDMP versus SOMP) point out that SOMP-based sequences provide slightly better classification accuracies, although the number of features required to produce optimal scores were smaller in the case of EDMP. However, further analysis of the results reveals important differences between the two considered classifiers. For example, the accuracy exhibited by SOMP-SID on directional features present in the Salinas A subscene [see Fig. 4(a)] is significantly higher than the one exhibited by EDMP-SID (it should be noted that the romaine lettuce is at different weeks since planting and with growth increasingly covering the soil, thus resulting in distinct spectral signatures at the different lettuce_romaine fields). From Table III, it is also clear that SOMP-SID outperforms EDMP-SID in the six Salinas A classes in terms of individual test accuracies, a fact that leads to a significant decrease in the AVE score associated to EDMP-SID with regard to the one

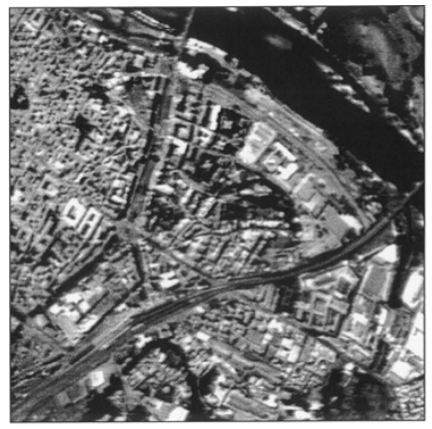

(a)

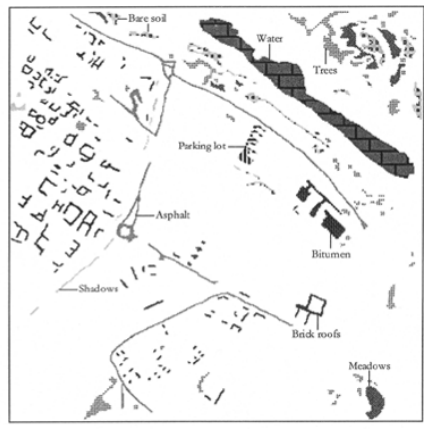

(b)
Fig. 6. (a) Band at $639 \mathrm{~nm}$ of a DAIS 7915 scene over Pavia city, Italy. (b) Available ground truth classes.

TABLE IV

INFORMATION CLASSES AND SAMPLES FOR EXPERIMENT 2 (DAIS 7915 PAVIA SCENE)

\begin{tabular}{lcc}
\hline Class & Training Samples & Test Samples \\
\hline Water & 114 & 4176 \\
Trees & 101 & 2444 \\
Asphalt & 85 & 1614 \\
Parking lot & 59 & 229 \\
Bitumen & 65 & 620 \\
Brick roofs & 106 & 2132 \\
Meadows & 62 & 1183 \\
Soil & 74 & 1401 \\
Shadows & 52 & 181 \\
\hline Total & 718 & 13979 \\
\hline
\end{tabular}

exhibited by SOMP-SID. This last item, which also applies to the SAD and HMMID distances, confirms the effectiveness of SOMP-based sequences in exploiting both the spectral and orientation information provided by directional features in the scene.

As a final comment, it should be noted that increasing the number of training samples to half of the available ground truth did not have a significant impact on the performance of EDMP- 


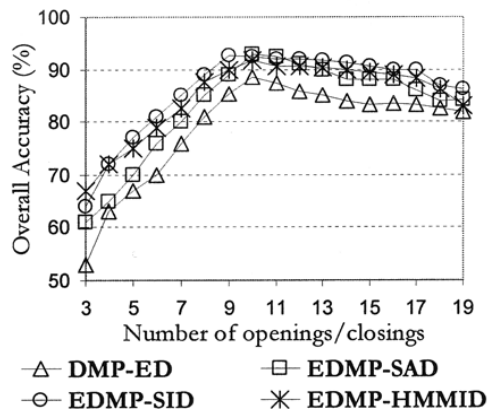

(a)

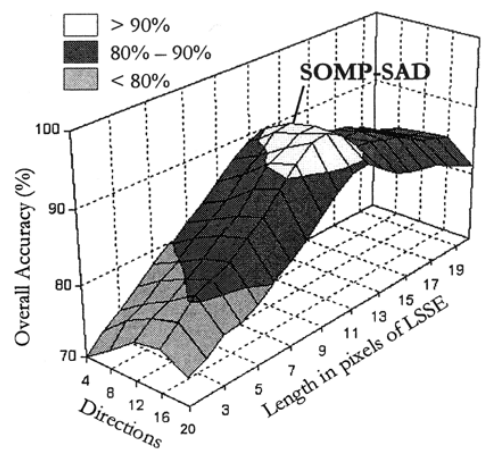

(d)

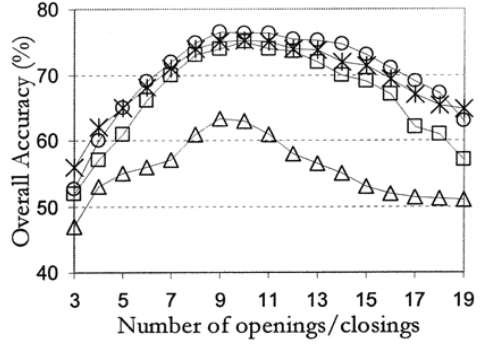

$\triangle$ OASF-ED

$\square-$ EOASF-SAD

(b)

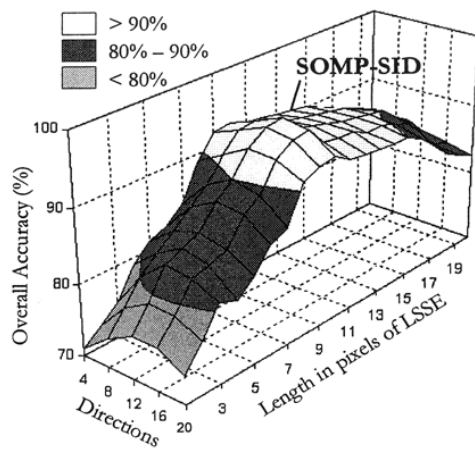

(e)

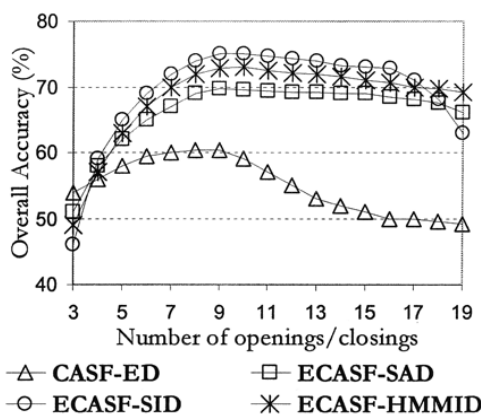

(c)

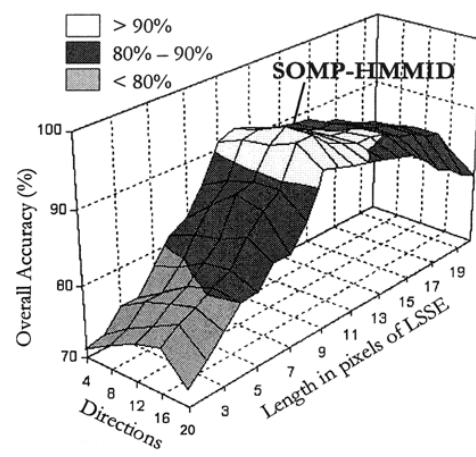

(f)

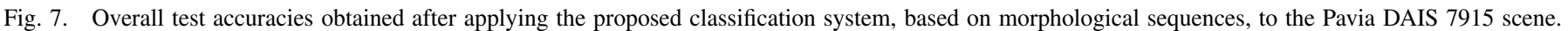
(a) DMP- and EDMP-based sequences. (b) OASF- and EOASF-based sequences. (c) CASF- and ECASF-based sequences. (d)-(f) SOMP-based sequences.

TABLE V

Dimension of FEATURe Vectors (DFV), Number of FEATURES (NOF) USED FOR ClassifiCATION AFTER DBFE FEATURE EXTRACTION, OVERALL (OA), Average (AVE) And Individual Test ACCuracies in Percentage Obtained After Applying the Proposed Classification System, Based on Morphological SEQuences With 10 Opening/Closing Operations, to the Pavia DAIS 7915 ScENE (SOMP-Based Features Were ObTained

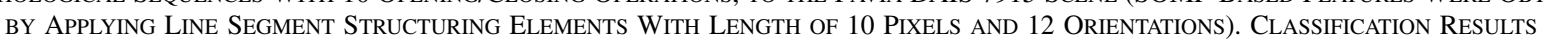
ObTAINED USING THE ORIGINAL SPECTRAL INFORMATION OF THE HyPERSPECTRAL SCENE ARE ALSO INCLUDED FOR COMPARISON

\begin{tabular}{|c|c|c|c|c|c|c|c|c|c|c|c|c|c|c|c|c|}
\hline Class & $\begin{array}{c}\text { Original } \\
\text { Spectral } \\
\text { Information }\end{array}$ & $\begin{array}{c}\text { DMP- } \\
\text { ED }\end{array}$ & $\begin{array}{l}\text { EDMP- } \\
\text { SAD }\end{array}$ & $\begin{array}{l}\text { EDMP- } \\
\text { SID }\end{array}$ & $\begin{array}{l}\text { EDMP. } \\
\text { HMMID }\end{array}$ & $\begin{array}{c}\text { OASF- } \\
\text { ED }\end{array}$ & $\begin{array}{c}\text { EOASF- } \\
\text { SAD }\end{array}$ & $\begin{array}{c}\text { EOASF- } \\
\text { SID }\end{array}$ & $\begin{array}{l}\text { EOASF- } \\
\text { HMMID }\end{array}$ & $\begin{array}{c}\text { CASF- } \\
\text { ED }\end{array}$ & $\begin{array}{l}\text { ECASF- } \\
\text { SAD }\end{array}$ & $\begin{array}{c}\text { ECASF- } \\
\text { SID }\end{array}$ & $\begin{array}{l}\text { ECASF- } \\
\text { HMMID }\end{array}$ & $\begin{array}{c}\text { SOMP- } \\
\text { SAD }\end{array}$ & $\begin{array}{l}\text { SOMP- } \\
\text { SID }\end{array}$ & $\begin{array}{l}\text { SOMP- } \\
\text { HMMID }\end{array}$ \\
\hline Water & 87.30 & 86.17 & 88.04 & 87.45 & 87.63 & 62.56 & 76.46 & 77.01 & 76.51 & 60.23 & 70.49 & 75.91 & 73.54 & 94.27 & 95.16 & 94.89 \\
\hline Trees & 94.64 & 97.62 & 97.94 & 97.58 & 97.12 & 68.44 & 75.81 & 76.36 & 76.12 & 58.85 & 69.58 & 74.48 & 74.61 & 96.68 & 97.21 & 96.94 \\
\hline Asphalt & 97.79 & 84.48 & 85.24 & 86.23 & 86.15 & 63.07 & 73.12 & 74.54 & 73.89 & 59.39 & 72.25 & 75.86 & 73.28 & 95.16 & 95.72 & 95.35 \\
\hline Parking lot & 83.82 & 81.93 & 82.15 & 81.97 & 81.17 & 67.23 & 71.63 & 71.87 & 72.34 & 63.11 & 68.44 & 69.54 & 71.02 & 90.85 & 91.14 & 90.76 \\
\hline Bitumen & 86.11 & 75.48 & 76.44 & 76.12 & 75.42 & 68.86 & 72.17 & 73.28 & 72.95 & 61.92 & 67.06 & 70.69 & 69.43 & 89.64 & 90.10 & 89.73 \\
\hline Brick roofs & 83.69 & 82.36 & 82.75 & 82.40 & 82.89 & 65.01 & 75.08 & 76.84 & 75.47 & 57.07 & 71.22 & 77.02 & 75.89 & 96.13 & 96.51 & 96.04 \\
\hline Meadows & 88.88 & 89.86 & 89.39 & 89.67 & 88.63 & 60.35 & 73.99 & 77.93 & 75.21 & 58.40 & 69.99 & 76.24 & 74.02 & 91.23 & 92.05 & 91.57 \\
\hline Soil & 79.85 & 84.68 & 85.21 & 84.87 & 85.38 & 62.11 & 72.65 & 75.87 & 73.67 & 60.98 & 66.80 & 74.49 & 70.54 & 92.45 & 92.77 & 92.81 \\
\hline Shadows & 89.64 & 92.81 & 92.64 & 92.50 & 92.44 & 61.19 & 77.24 & 79.03 & 78.76 & 58.03 & 67.79 & 79.85 & 72.31 & 95.06 & 95.69 & 95.14 \\
\hline DFV & 40 & 20 & 20 & 20 & 20 & 20 & 20 & 20 & 20 & 20 & 20 & 20 & 20 & 240 & 240 & 240 \\
\hline NOF & 13 & 11 & 11 & 12 & 12 & 9 & 10 & 11 & 11 & 8 & 9 & 11 & 10 & 16 & 18 & 17 \\
\hline $\mathrm{OA}$ & 88.65 & 89.75 & 90.94 & 90.71 & 90.65 & 63.21 & 75.02 & 76.49 & 75.94 & 59.93 & 69.63 & 75.14 & 73.28 & 94.48 & 95.57 & 95.26 \\
\hline AVE & 87.96 & 86.14 & 86.64 & 86.53 & 86.31 & 64.31 & 74.23 & 75.85 & 74.99 & 59.77 & 69.29 & 74.89 & 72.73 & 93.49 & 94.03 & 89.75 \\
\hline
\end{tabular}

and SOMP-based methods; the observed increase was always below $1 \%$ in terms of OA. A more significant improvement was observed when the original spectral information was used. Specifically, the OA increased to $92.14 \%$, which is below the OAs obtained by using EDMP- and SOMP-based classifiers with less training samples. This fact reveals that the proposed morphological methods can perform well with limited training samples. In order to fully assess the effectiveness of the proposed approaches, we must take into account that large and spectrally homogeneous regions dominate the Salinas scene. As a result, a very simple behavior of morphological profiles was generally observed, where each feature had only one or two significant derivative maxima. Complex scenarios such as urban environments are, however, characterized by small directional features with several significant derivative maxima and nested regions. As a result, further experimentation using real hyperspectral data collected over urban areas is rather pertinent.

\section{B. Experiment 2: DAIS 7915 Data Over Pavia City, Italy}

For this trial, data from the DAIS 7915 airborne imaging spectrometer of DLR were used. The data were collected at $1500 \mathrm{~m}$ over the city of Pavia, Italy. The flight altitude was chosen as the lowest available for the airplane, which resulted in a 5-m spatial resolution [43]. Fig. 6(a) shows a $400 \times 400$ pixel scene collected at $639 \mathrm{~nm}$ by the DAIS 7915 imaging spectrometer, which reveals a dense residential area on one side of the river, as well as open areas and meadows on the other side. Ground truth information is available for several 
areas of the scene [see Fig. 6(b)], comprising the following land-cover classes: 1) Water; 2) Trees; 3) Asphalt; 4) Parking lot; 5) Bitumen; 6) Brick roofs; 7) Meadows; 8) Bare soil; 9) Shadows. Following a previous research study on this scene [22], we take into account only 40 spectral bands of reflective energy, and thus skip thermal infrared bands and middle infrared bands above $1958 \mathrm{~nm}$ because of low SNR in those bands. A DBFE-based classifier was trained with a variety of morphological sequences, constructed for a sample of 5\% of the pixels from the ground truth of the nine classes in Fig. 6(b) where the information classes and training and test samples are listed in Table IV.

A summary of the overall classification accuracies obtained by the above system for test data as a function of the number of opening/closing operations for multichannel (EDMP, EOASF, and ECASF) and monochannel (DMP, OASF, and CASF) morphological sequences is shown in Fig. 7(a)-(c). From this figure, it is clear that classification based on EDMP and DMP sequences was more accurate than classification based on EOASF/ECASF, and on OASF/CASF. In all cases, the SID distance was found to be the most effective in characterizing spectral features. A maximum in overall classification accuracy was generally observed when the number of opening/closing operations was set to 10 . It is also interesting to note that the overall accuracies for the EDMP-based sequences did not improve much those found using DMP-ED sequences in this experiment. This fact provides an objective confirmation of our introspection: that scale-orientation information is required in order to complement spectral information when complex image scenes are to be analyzed.

Fig. 7(d)-(f) plots test classification accuracies obtained by SOMP sequences as a function of the length in pixels (scale) and number of directions (orientation). The difference from Experiment 1 is that much more improvement was achieved as the number of directions was increased. A probable reason for this improvement is that the DAIS 7915 scene contains proportionally more straight lines with arbitrary orientations and nested regions than the AVIRIS scene. However, when the number of directions was very high, a loss in classification performance was generally observed. With the above results in mind, we use line segment SEs with $p=10$ pixels in length and 12 orientations to create SOMP-based sequences in this experiment. As depicted in Table V, SOMP-based approaches clearly outperformed their EDMP-based counterparts. However, in terms of both OA and AVE accuracies, using the feature set consisting of EDMP-based sequences gave very similar results to the cases when DMP-ED and the original spectral information in the hyperspectral image were used. Interestingly, SOMP-based profiles produced very accurate classification results for the classes dominated by small directional features (Asphalt, Brick roofs, and Shadows). The incorporation of scale-orientation information also helped characterize large oriented objects (Water) and classes given by a combination of homogeneous regions and directional features (Bitumen, Parking lot, Bare soil). The above results were also observed when the number of training samples was increased to half of the available ground truth. Specifically,
SOMP achieved at least $96 \%$ OA for the three considered distances and outperformed all the other approaches including the spectral information, which achieved $92.79 \%$ OA.

In conclusion, we can state that experiments have demonstrated that EDMPs and SOMPs can work efficiently at local and global scales. This success is achieved by the fact that they provide a final classification output that is coherent in both spectral and spatial terms for a complex, real-world analysis scenario. Results in this section also point to the importance of having a system that can resolve both scale- and orientation-based spectral variations due to the integration of spatial/spectral information. This significance may also lead to new fields of application. For instance, in certain parts of the Pavia scene we can appreciate a more regular urban structure. Scale-orientation information may not be fully required in order to characterize these features. However, EDMPs alone may be unreliable when used for the accurate modeling of other areas in the scene where the shape and location of buildings is more irregular. This emphasizes the possibility of combining both approaches in order to solve problems related to city classification.

\section{CONCLUSION AND Future WORK}

This paper has described new morphological filtering techniques for hyperspectral image data analysis that take into account both the spatial and spectral information simultaneously. The considered approaches were: 1) extended differential morphological profiles; 2) extended alternated sequential filters; and 3) scale-orientation morphological profiles. The above methods were used in this paper to build feature vectors for supervised classification using DBFE for neural networks. Experimental results using two real hyperspectral datasets have demonstrated that morphological operations, extended to multichannel imagery by using well-known spectral-based distance metrics such as SAD, SID, and HMMID, provide an excellent tool for classification purposes. In addition, morphological operations preserve the relevant spatial/spectral information that allows for the separation of classes. Although the test data were different in many ways (e.g., application areas, spatial, and spectral resolution, radiance/reflectance data and sensor SNR), high overall accuracies were achieved for both datasets. In particular, feature extraction methods based on multichannel differential morphological operations were more effective than other approaches that consider spatial and spectral features separately. It was also found in the experiments that the incorporation of scale-orientation information to morphological profiles allows for accurate modeling of hyperspectral image scenes dominated by complex image structures, such as those present in urban areas.

As with any new approach, there are some unresolved issues that may present challenges over time. Specifically, the proposed techniques are particularly well suited to separate slightly different spectral features that are clearly distinct in the spatial domain. However, in order to associate together features with very similar spectral properties but different spatial properties, further work is still needed. In such cases, joint spatial-spectral classifiers may need to be replaced by more spectrally guided 
techniques. A drawback in the proposed approaches has to do with the need to heed a range of morphological filters with increasing scale and orientation features, which may result in a heavy computational burden when processing high-dimensional data. This phenomenon is particularly important in the case of images with large and spectrally homogeneous regions. In order to empower the proposed techniques with near real-time capabilities, we currently experiment with their efficient implementation on high-performance parallel computing architectures such as field-programmable gate arrays and Beowulf-type clusters.

\section{ACKNOWLEDGMENT}

The authors gratefully thank P. Gamba for providing the DAIS 7915 data over Pavia, Italy, along with the training and test sets. The authors would also like to thank J. A. Gualtieri for his valuable comments and also for his presentation of a preliminary version of this research at the IEEE Workshop on Advances in Techniques for Analysis of Remotely Sensed Data, a workshop honoring Prof. D. A. Landgrebe's outstanding career that has served as a guide and inspiration to the authors. In addition, the authors would like to express their gratitude to C.-I Chang, R. O. Green, and J. C. Tilton for their support to this research, and to J. Chanussot for some helpful discussions on multichannel morphological operations. The authors greatly appreciate the suggestions and comments by the anonymous reviewers and the linguistic revision of this paper by A. Curado (Department of English at UEX).

\section{REFERENCES}

[1] D. A. Landgrebe, Signal Theory Methods in Multispectral Remote Sensing. Hoboken, NJ: Wiley, 2003.

[2] R. O. Green et al., "Imaging spectroscopy and the Airborne Vsible/Infrared Imaging Spectrometer (AVIRIS)," Remote Sens. Environ., vol. 65, pp. 227-248, 1998.

[3] C.-I Chang, Hyperspectral Imaging: Techniques for Spectral Detection and Classification. New York: Kluwer/Plenum, 2003.

[4] X. Jia, J. A. Richards, and D. E. Ricken, Remote Sensing Digital Image Analysis: An Introduction. Berlin, Germany: Springer-Verlag, 1999.

[5] L. Jimenez and D. A. Landgrebe, "Supervised classification in high-dimensional space: Geometrical, statistical, and asymptotical properties of multivariate data," IEEE Trans. Syst., Man, Cybern. C, vol. 28, pp. 39-54, 1998.

[6] D. W. Scott, "The curse of dimensionality and dimension reduction," in Multivariate Density Estimation: Theory, Practice, and Visualization. New York: Wiley, 1992, ch. 7, pp. 195-217.

[7] S. Kaewpijit, J. Le Moigne, and T. El-Ghazawi, "Automatic reduction of hyperspectral imagery using wavelet spectral analysis," IEEE Trans. Geosci. Remote Sens., vol. 41, no. 4, pp. 863-871, Apr. 2003.

[8] F. Tsai and W. Philpot, "Derivative analysis of hyperspectral data," Remote Sens. Environ., vol. 66, pp. 41-51, 1998.

[9] L. M. Bruce, C. H. Koger, and J. Li, "Dimensionality reduction of hyperspectral data using discrete wavelet transform feature extraction," IEEE Trans. Geosci. Remote Sens., vol. 40, no. 10, pp. 2331-2338, Oct. 2002.

[10] V. Madhok and D. Landgrebe, "Spectral-spatial analysis of remote sensing data: An image model and a procedural design," Ph.D. dissertation, School of Elect. Eng. Comput. Sci., Purdue Univ., Lafayette, IN, 1998.

[11] A. Plaza, P. Martinez, R. Perez, and J. Plaza, "Spatial/spectral endmember extraction by multidimensional morphological operations," IEEE Trans. Geosci. Remote Sens., vol. 40, no. 9, pp. 2025-2041, Sep. 2002.
[12] A. Plaza, "Development, validation, and testing of a new morphological method for hyperspectral image analysis that integrates spatial and spectral information," Ph.D. dissertation, Comput. Sci. Dept., Univ. Extremadura, Cáceres, Spain, 2002.

[13] J. Serra, Image Analysis and Mathematical Morphology. New York: Academic, 1982.

[14] P. Soille, Morphological Image Analysis: Principles and Applications, 2nd ed. Berlin, Germany: Springer-Verlag, 2003.

[15] G. Matheron and J. Serra, "The birth of mathematical morphology," in Proc. 6th Intl. Symp. Mathematical Morphology, H. Talbot and R. Beare, Eds., Sydney, Australia, 2002, pp. 1-16.

[16] S. R. Sternberg, "Grayscale morphology," Comput. Vision Graphics Image Process., vol. 35, pp. 333-355, 1986.

[17] J. Goutsias and H. Heijmans, "Fundamenta morphologicae mathematicae," Fund. Inf., vol. 41, pp. 1-30, 1999.

[18] C. Ronse and H. Heijmans, "The algebraic basis of mathematical morphology: II. Openings and closings," Comput. Vis., Graph., Image Process.: Image Understand., vol. 54, pp. 74-97, 1991.

[19] M. Pesaresi and J. A. Benediktsson, "A new approach for the morphological segmentation of high resolution satellite imagery," IEEE Trans. Geosci. Remote Sens., vol. 39, no. 2, pp. 309-320, Feb. 2001.

[20] M. Pesaresi and I. Kannellopulos, "Detection of urban features using morphological based segmentation and very high resolution remotely sensed data," in Machine Vision and Advanced Image Processing in Remote Sensing, I. Kanellopoulos, G. G. Wilkinson, and T. Moons, Eds, Berlin, Germany: Springer-Verlag, 1999.

[21] J. A. Benediktsson, M. Pesaresi, and K. Arnason, "Classification and feature extraction for remote sensing images from urban areas based on morphological transformations," IEEE Trans. Geosci. Remote Sens., vol. 41, no. 9, pp. 1940-1949, Sep. 2003.

[22] J. A. Palmason, J. A. Benediktsson, and K. Arnason, "Morphological transformations and feature extraction for urban data with high spectral and spatial resolution," in Proc. IGARSS, Toulouse, France, 2003.

[23] J. Chanussot, J. A. Benediktsson, and M. Pesaresi, "On the use of morphological alternated sequential filters for the classification of remote sensing images from urban areas," in Proc. IGARSS, Toulouse, France, 2003.

[24] J. Goutsias, H. Heijmans, and K. Sivakumar, "Morphological operators for image sequences," Comput. Vis. Image Understand, vol. 62, pp. 326-346, 1995.

[25] G. Louverdis, M. I. Vardavoulia, I. Andreadis, and Ph. Tsalides, "A new approach to morphological color processing," Pattern Recognit., vol. 35, pp. 1733-1741, 2002.

[26] I. Pitas and C. Kotropoulos, "Multichannel L filters based on marginal data ordering," IEEE Trans. Signal Process., vol. 42, pp. 2581-2595, 1994.

[27] K. Tang, J. Astola, and Y. Neuvo, "Nonlinear multivariate image filtering techniques," IEEE Trans. Image Process., vol. 4, no. 6, pp. 788-798, Jun. 1995.

[28] A. Plaza, P. Martinez, R. Perez, and J. Plaza, "Spatial/spectral analysis of hyperspectral image data," presented at the IEEE Workshop Advances in Techniques for Remotely Sensed Data Analysis, Greenbelt, MD, 2003.

[29] - "Hyperspectral image analysis by scale-orientation morphological profiles," in Proc. SPIE Conf. Image and Signal Process. for Remote Sensing, Barcelona, Spain, 2003.

[30] P. Soille and M. Pesaresi, "Advances in mathematical morphology applied to geoscience and remote sensing," IEEE Trans. Geosci. Remote Sens., vol. 40, no. 9, pp. 2042-2055, Sep. 2002.

[31] P. Soille and H. Talbot, "Directional morphological filtering," IEEE Trans. Pattern Anal. Machine Intell., vol. 23, no. 11, pp. 1313-1329, Nov. 2001.

[32] N. Keshava and J. F. Mustard, "Spectral unmixing," IEEE Signal Process. Mag., vol. 19, no. 1, pp. 44-57, Jan. 2002.

[33] C.-I Chang, "An information theoretic-based approach to spectral variability, similarity, and discriminability for hyperspectral image analysis," IEEE Trans. Inform. Theory, vol. 46, no. 5, pp. 1927-1932, Aug. 2000.

[34] Q. Du and C.-I Chang, "Hidden Markov model approach to spectral analysis for hyperspectral imagery," Opt. Eng., vol. 40, pp. 2277-2284, 2001.

[35] J. Crespo, J. Serra, and R. Schafer, "Theoretical aspects of morphological filters by reconstruction," Signal Process., vol. 47, pp. 201-225, 1995.

[36] I. Bloch, "Geodesic balls in a fuzzy set and fuzzy geodesic mathematical morphology," Pattern Recognit., vol. 33, pp. 897-905, 2000.

[37] A. Müeller, A. Hausold, and P. Strobl, "HySens-DAIS/ROSIS imaging spectrometers at DLR," in Proc. VIII SPIE Intl. Symp. Remote Sensing, Toulouse, France, 2001. 
[38] M. Lybanon, "Maltese front variability from satellite observations based on automated detection," IEEE Trans. Geosci. Remote Sens., vol. 34, no. 5, pp. 1159-1165, Sep. 1996.

[39] C. Lee and D. A. Landgrebe, "Feature extraction based on decision boundaries," IEEE Trans. Pattern Anal. Machine Intell., vol. 15, no. 4, pp. 388-400, Apr. 1993.

[40] J. A. Benediktsson, J. R. Sveinsson, and K. Arnason, "Classification and feature extraction of AVIRIS data," IEEE Trans Geosci. Remote Sens., vol. 33, no. 6, pp. 1194-1205, Nov. 1993.

[41] C. Lee and D. A. Landgrebe, "Decision boundary feature extraction for neural networks," IEEE Trans. Neural Networks, vol. 8, no. 1, pp. 75-83, Jan. 1997.

[42] J. A. Gualtieri, S. R. Chettri, R. F. Cromp, and L. F. Johnson, "Support vector machine classifiers as applied to AVIRIS data," in Summaries of the 8th JPL Airborne Earth Science Workshop, R. O. Green, Ed., 1999, JPL Pub. 99-17, pp. 217-227.

[43] F. Dell'Aqua, P. Gamba, and A. Ferrari, "Exploiting spatial and spectral information for classifying hyperspectral data in urban areas," in Proc. IGARSS, Toulouse, France, 2003.

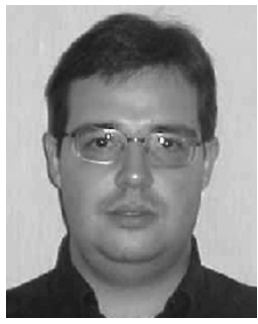

Antonio Plaza (M’05) received the M.S. and Ph.D. degrees in computer science from the University of Extremadura, Cáceres, Spain, in 1997 and 2002, respectively.

$\mathrm{He}$ is currently an Associate Professor with the Computer Science Department, University of Extremadura, since 2000, where he was a Research Associate and Assistant Professor from 1997 to 1999. He was also a Visiting Researcher with the Remote Sensing Signal and Image Processing Laboratory, University of Maryland Baltimore County, and also with the Applied Information Sciences Branch, Goddard Space Flight Center, Greenbelt, MD, and the AVIRIS Group, Jet Propulsion Laboratory, Pasadena, CA. His main research interests span computer vision, image processing, pattern recognition, and development and efficient implementation of hyperspectral image analysis algorithms on massively parallel computing facilities and VLSI hardware-based computer architectures. He has authored or coauthored more than 70 publications including journal papers and conference proceedings.

Dr. Plaza is currently serving as a reviewer for the IEEE TRANSACTIONS ON GEOSCIENCE AND Remote Sensing, IEEE TRANSACtions ON IMAGE PROCESSING, IEEE TRANSACTIONS ON PARALLEL AND DistRIBUTED SySTEMS, IEEE TRANSACTIONS ON INFORMATION THEORY, and IEEE GEOSCIENCE AND REMOTE SENSING LETTERS.

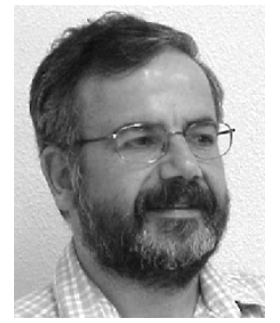

Pablo Martínez received the Ph.D. degree in physics from the University of Granada, Granada, Spain, in 1992.

He has been a Professor of computer science with the University of Extremadura, Cáceres, Spain, since 1985 . He is currently the Head Scientist of the Neural Networks and Signal Processing Group (GRNPS). He has held Visiting Researcher positions at the Applied Information Sciences Branch, Goddard Space Flight Center, Greenbelt, MD, the Department of Electrical Engineering, University of Maryland, College Park, and the AVIRIS Group, Jet Propulsion Laboratory, Pasadena, CA. His main research interests include remote sensing, digital image analysis, hardware-based architectures, operating systems management and configuration, and neural-network-based pattern recognition.

Dr. Martínez has been a reviewer for the IEEE TRANSACTIONS ON GEOSCIENCE AND REMOte SENSING.

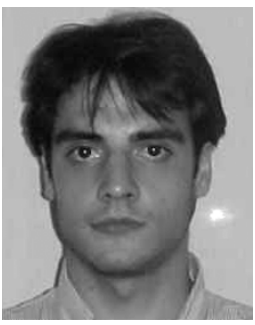

Javier Plaza received the M.Sc. degree in computer science from the University of Extremadura, Cáceres, Spain, in 2002, where he is currently pursuing the Ph.D. degree.

$\mathrm{He}$ is currently an Assistant Professor in the Computer Science Department, University of Extremadura. His current research work is focused on the development of efficient implementations of nonlinear mixture model-based algorithms for abundance estimation of materials in hyperspectral scenes. He is also involved in the design and configuration of commodity cluster computing architectures for high-performance hyperspectral analysis. Other major research interests include development of quantitative and comparative applications for remote sensing, and the study of configuration and training of neural network architectures for hyperspectral image analysis.

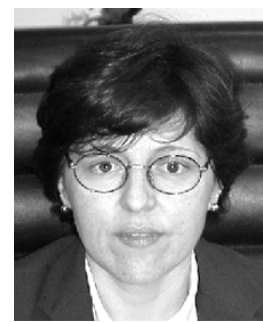

Rosa Pérez received the M.S. degree in mathematics from the University of Extremadura, Cáceres, Spain, in 1985 , and the Ph.D. degree in computer science from the Polytechnic University of Madrid, Madrid, Spain, in 1995.

She is currently a Professor of computer science in the Computer Science Department, University of Extremadura, Cáceres, where she has been since 1985. Her main research interests include neural networks, systolic array- and FPGA-based design, pattern recognition, and signal processing. 